For Unlimited Distribution

\title{
Project Title: ACTIVE FLOW CONTROL ON BIDIRECTIONAL ROTORS FOR TIDAL MHK APPLICATIONS
}

Final Technical Report

DOE Award Number: DE-EE0004568

Recipient: The Regents of the University of California DUNS 04-712-0084

Principal Investigator:

C.P. van Dam

Professor and Department Chair cpvandam@ucdavis.edu

Consortium/Teaming

Members: $n / a$

Submitted by: Henry Shiu

Research Engineer

hjshiu@ucdavis.edu 


\section{EXECUTIVE SUMMARY}

A marine and hydrokinetic (MHK) tidal turbine extracts energy from tidal currents, providing clean, sustainable electricity generation. In general, all MHK conversion technologies are confronted with significant operational hurdles, resulting in both increased capital and operations and maintenance (O\&M) costs. To counter these high costs while maintaining reliability, MHK turbine designs can be simplified.

Prior study found that a tidal turbine could be cost-effectively simplified by removing blade pitch and rotor/nacelle yaw. Its rotor would run in one direction during ebb and then reverse direction when the current switched to flood. We dubbed such a turbine a bidirectional rotor tidal turbine (BRTT). The bidirectional hydrofoils of a BRTT are less efficient than conventional hydrofoils and capture less energy, but the elimination of the pitch and yaw systems were estimated to reduce levelized cost of energy by $7.8 \%-9.6 \%$ [1]

In this study, we investigated two mechanisms for recapturing some of the performance shortfall of the BRTT. First, we developed a novel set of hydrofoils, designated the yy series, for BRTT application. Second, we investigated the use of active flow control via microtabs. Microtabs are small deployable/retractable tabs, typically located near the leading or trailing edge of an air/hydrofoil with height on the order of the boundary layer thickness ( $1 \%-2 \%$ of chord). They deploy approximately perpendicularly to the foil surface and, like gurney flaps and plain flaps, globally affect the aerodynamics of the airfoil. By strategically placing microtabs and selectively deploying them based on the direction of the inflow, performance of a BRT'T rotor can be improved while retaining bidirectional operation.

The yy foils were computationally designed and analyzed. They exhibited better performance than the baseline bidirectional foil, the ellipse. For example, the yyb07cn-180 had $14.7 \%$ higher $(1 / \mathrm{d})_{\max }$ than an ellipse of equal thickness. The yyb07cn family also had higher $\mathrm{c}_{\mathrm{p} \text {,min }}$ than equivalently thick ellipses, indicating less susceptibility to cavitation.

Microtabs applied on yy foils demonstrated improved energy capture. A series of variable speed and constant speed rotors were developed with the yyb07cn family of hydrofoils. The constant speed yyb07cn rotor (yy-B02-R $s$, opt) captured $0.45 \%$ more energy than the equivalent rotor with ellipses (e-B02-R cs,opt). With microtabs deployed (yy $\mu \mathrm{t}-\mathrm{B} 02-\mathrm{R} c s, o p t)$, the energy capture increase over the rotor with ellipses was $1.05 \%$. Note, however, that microtabs must be applied judiciously to bidirectional foils. On the $18 \%$ thick ellipse, performance decreased with the addition of microtabs.

Details of hydrofoil performance, microtab sizing and positioning, rotor configurations, and revenue impacts are presented herein. 


\section{TABLE OF CONTENTS}

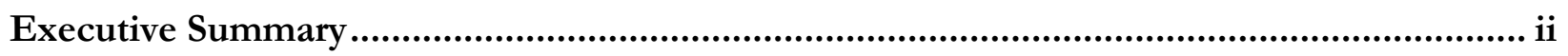

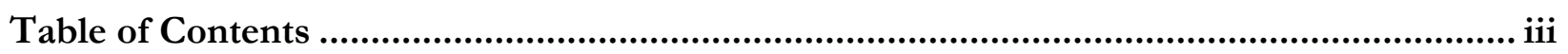

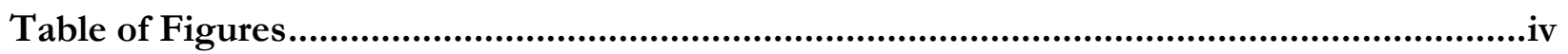

Table of Tables ................................................................................................................

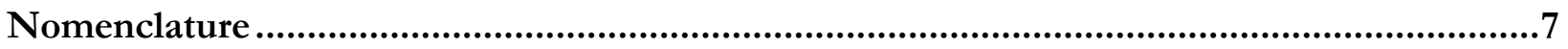

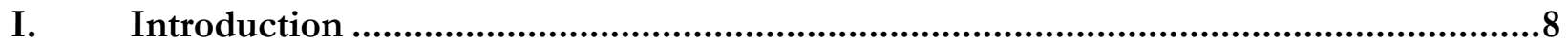

II. Project Activities: Hydrofoil and Rotor Design and Analysis ..................................... 11

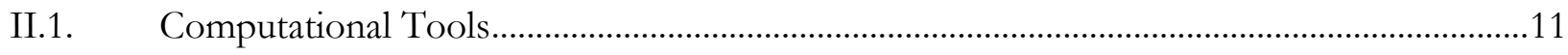

II.2. Hydrofoils for Bidirectional Tidal Turbines .....................................................................11

II.2.a. Elliptical Hydrofoils and Other Initial Hydrofoil Candidates ............................................11

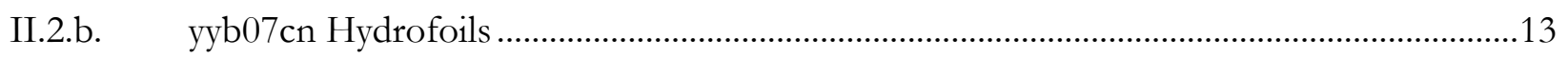

II.2.c. $\quad$ Microtabs on Ellipses and yyb07cn Hydrofoils ..............................................................14

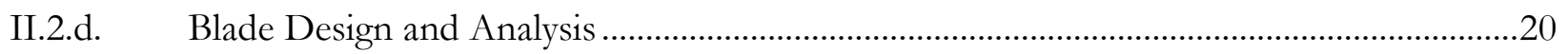

III. Energy and Economic Analysis...........................................................................22

III.1. Characteristic Current Speeds ………………………………………………………....22

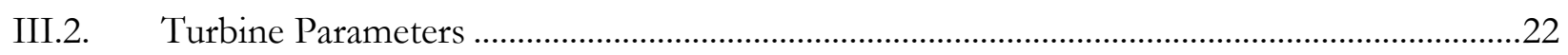

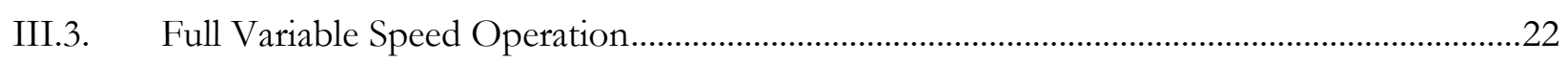

III.4. Constant Speed Operation with Stall REgulation.................................................................24

IV. Comparison of Accomplishments with Initial Goals and Objectives .........................29

IV.1. Summary of accomplishments and Key Findings................................................................2.

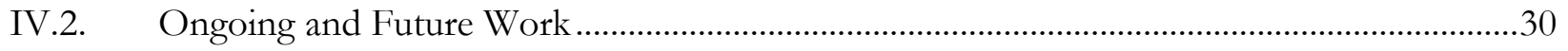

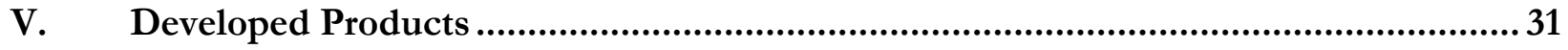

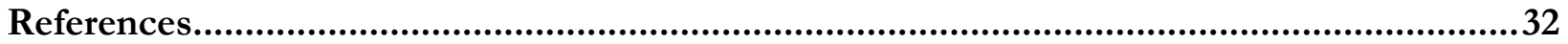




\section{TABLE OF FIGURES}

Figure I.1. Rendering of Voith Hydro Ocean Current Technology's BRTT. ....................................8

Figure I.2. Blade cross-section of a BRTT with microtabs. $\mathrm{V}_{\mathrm{c}}$ is the current velocity. $\mathrm{F}_{\mathrm{TAN}}$ is the tangential component of the hydrodynamic forces produced by the blade; this is the force component that drives the rotor. In the upper-left, the microtabs are retracted. In the upper-right, the microtabs are also retracted, but the current is reversed, driving the rotor in the opposite direction. In the lower pair of images, the microtabs on the high pressure side of the blade are deployed (as detailed in the callout), increasing the

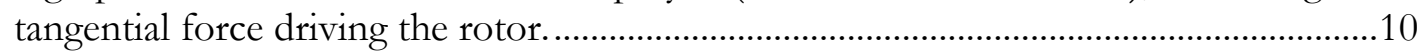

Figure II.1. Ellipse and m67-018 foils, thickness of 18\% of chord...................................................12

Figure II.2. Enlargement of ellipse and m67-018 foils, thickness of 18\% chord.............................12

Figure II.3. Comparison of performance of ellipse, m67-018, yy636-cn-18, and yy636-c03-18. $(\mathrm{t} / \mathrm{c})_{\max }=18 \%, \mathrm{Re}=1.5 \times 10^{6}, \mathrm{M}_{\infty}=0.2$, fully turbulent. OVERFLOW.............................13

Figure II.4. Hydrodynamic performance of the elliptical and yyb07cn hydrofoil families. Each family has hydrofoils of thickness $18 \% \mathrm{c}, 24 \% \mathrm{c}$, and $35 \% \mathrm{c}$. $\mathrm{Re}=1.5 \times 10^{6}, \mathrm{M}_{\infty}=0.2$, fully

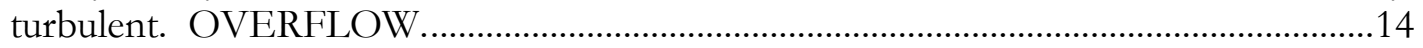

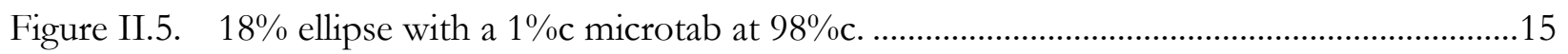

Figure II.6. Performance of the $18 \%$ ellipse with microtab ( $\mu$ tab) on the high pressure surface near the trailing edge. Microtab height: $1 \%$ c. Microtab thickness: $0.2 \%$ c. Re $=1.5 \times 10^{6}$,

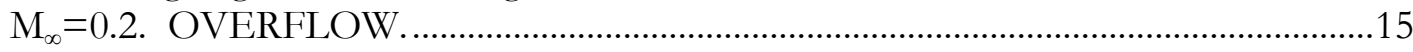

Figure II.7. Velocity magnitude of the $18 \%$ ellipse at $\alpha=0^{\circ}, \mathrm{Re}=1.5 \times 10^{6}, \mathrm{M}_{\infty}=0.2$, fully turbulent. OVERFLOW. a. No microtab. b. Microtab at $94 \%$ with $1 \%$ c height. .16

Figure II.8. Velocity magnitude of the $18 \%$ ellipse at $\alpha=12^{\circ}, \mathrm{Re}=1.5 \times 10^{6}, \mathrm{M}_{\infty}=0.2$, fully turbulent. OVERFLOW. a. No microtab. b. Microtab at $98 \%$ c with $1 \%$ c height; note vortex shedding.....

Figure II.9. Hydrodynamic performance of the yyb07cn-180 with a microtab on the high pressure surface. Microtab height: 1.0\%c. Microtab location: 94\%c - 99\%c. OVERFLOW, $\mathrm{Re}=1.5 \times 10^{6}, \mathrm{M}_{\infty}=0.2$, fully turbulent.

Figure II.10. Hydrodynamic performance of the yyb07cn-180 with a microtab on the high pressure surface. Microtab height: $1.5 \%$ c. Microtab location: $94 \%$ c $-99 \%$ c. OVERFLOW, $\mathrm{Re}=1.5 \times 10^{6}, \mathrm{M}_{\infty}=0.2$, fully turbulent. .

Figure II.11. Hydrodynamic performance of the yyb07cn-180 with a microtab on the high pressure surface. Microtab height: 2.0\%c. Microtab location: 94\%c - 99\%c. OVERFLOW, $\mathrm{Re}=1.5 \times 10^{6}, \mathrm{M}_{\infty}=0.2$, fully turbulent.

Figure II.12. Hydrodynamic performance of the yyb07cn-180 with a microtab on the high pressure surface. Microtab height: $1.0 \%$ c, $1.5 \%$ c, $2.0 \%$ c. Microtab location: $97 \%$ c, $98 \%$ c. OVERFLOW, $\mathrm{Re}=1.5 \times 10^{6}, \mathrm{M}_{\infty}=0.2$, fully turbulent.

Figure II.13. $\mathrm{C}_{\mathrm{P}}$-TSR curves of three-bladed rotors using the e-B02 (using elliptical hydrofoils), yyB02 (using yyb07cn hydrofoils), yy $\mu \mathrm{t}-\mathrm{B} 02$ (using yyb07cn hydrofoils and designed for 
outboard microtabs; results are shown with microtabs deployed and retracted) blades...

Figure III.1. Current speed probability distributions of five prospective tidal power sites. A Rayleigh-based distribution with an equal mean of $0.87 \mathrm{~m} / \mathrm{s}$ is included. 22

Figure III.2. Power curves of variable speed rotors with elliptical hydrofoils (e-B02-Rvs), yy hydrofoils (yy-B02-R $v s$ ), yy hydrofoils with microtabs deployed (yy $\mu \mathrm{t}-\mathrm{B} 02-\mathrm{R} v s$ ), and yy hydrofoils with microtabs retracted (yy $\mathrm{t} \mathrm{t}-\mathrm{B} 02-\mathrm{R} v s)$. In the left plot (a), the curves appear to almost overlap. In the right plot (b), a zoom-in to Region II shows the performance differences between the rotors.

Figure III.3. Power curve of e-B02-Rcs,opt, a stall-regulated rotor with a rated power of $60 \mathrm{~kW}$. The dashed line represents power production without a prescribed cut-out.

Figure III.4. Power curves of yy-B02-Rcs,opt and yy-B02-Rstall, stall-regulated rotors with a rated power of $60 \mathrm{~kW}$. The dashed line for the yy-B02-R $c$, opt represents power production without a prescribed cut-out.

Figure III.5. Power curves of yy $\mu \mathrm{t}-\mathrm{B} 02-\mathrm{R} c s$, opt-dep (optimized for energy capture with microtabs deployed), yy $\mu t-B 02-R c s$, fail (optimized for energy capture with microtabs deployed, but with microtabs stuck in the retracted position), and yy $\mu$ t-B02-Rcs,opt (optimized for energy capture with microtabs optimally deployed and retracted). These are stallregulated rotors with a rated power of $60 \mathrm{~kW}$. The dashed lines represent performance without prescribed cut-out. 


\section{TABLE OF TABLES}

Table III-1. Capacity factors of variable speed rotors (-B02-Rvs) assuming power regulation to rated power, cut-out at $3 \mathrm{~m} / \mathrm{s}$, and a Rayleigh distribution with a mean speed of 0.87

$\mathrm{m} / \mathrm{s}$.

Table 0-1. Capacity factors of constant speed rotors (-B02-Rcs) assuming prescribed cut-out to prevent exceeding rated power and a Rayleigh distribution with a mean speed of 0.87

$\mathrm{m} / \mathrm{s}$.

Table III-3. Comparison of energy capture/revenue between various constant speed rotor configurations. Changes in annual revenue are presented for a range of energy prices. 


\section{NOMENCLATURE}

BEM Blade element momentum theory

BRTT Bidirectional rotor tidal turbine

c Chord

CFD Computational fluid dynamics

$c_{1} \quad$ Lift coefficient

$\mathrm{c}_{1, \max } \quad$ Maximum lift coefficient

$\mathrm{C}_{\mathrm{P}} \quad$ Power coefficient

$\mathrm{C}_{\mathrm{P}, \max } \quad$ Maximum power coefficient

$\mathrm{c}_{\mathrm{p}, \min } \quad$ Minimum surface pressure coefficient

1/d Lift to drag ratio

$(\mathrm{l} / \mathrm{d})_{\max } \quad$ Maximum lift to drag ratio

MHK Marine-hydrokinetc

$\mathrm{M}_{\infty} \quad$ Freestream Mach number

$\mathrm{R} \quad$ Rotor radius

RaNS Reynolds-average Navier-Stokes

Re Reynolds number

TRL Technology readiness level

TSR Tip speed ratio

t/c thickness to chord ratio

$(\mathrm{t} / \mathrm{c})_{\max }$ Maximum thickness to chord ratio

$\lambda \quad$ Tip speed ratio

$\lambda_{\text {opt }} \quad$ Optimum tip speed ratio

$\mu \mathrm{t} \quad$ Microtab

$\mu$ tab Microtab 


\section{INTRODUCTION}

Marine and hydrokinetic ( $\mathrm{MHK}$ ) resources are vast and largely untapped, with potential to become a significant, clean, sustainable source of electricity generation. There is a diverse array of MHK conversion devices, with both novel designs and adaptations of established technologies. Currentdriven rotor-based turbines are an example of the latter. Most have borrowed heavily from the wind power industry, using features from what has emerged as the standard, most economical turbine configuration: variable pitch blades, a horizontal-axis rotor coupled to a variable speed generator, and a yawing nacelle.

While wind and MHK power are analogous in many ways, there are significant differences. Underwater and potentially far from land, MHK turbines are much more difficult to access once installed. In marine applications, they operate in a corrosive medium. Relatively high pressure seals are required to protect machine interiors at depth and against wave action, particularly at the junctures of moving parts. There is greater risk of fouling, from settlement and subsequent growth of aquatic organisms on machine surfaces and from entanglement with aquatic vegetation or manmade debris. All these factors drive up both initial capital costs and operations and maintenance $(\mathrm{O} \& \mathrm{M})$ costs.

These issues can be countered while maintaining reliability by simplifying turbine designs. For tidal sites with only two prevailing current directions (in $180^{\circ}$ opposition to each other), pitch and yaw mechanisms can be completely eliminated with a bidirectional rotor that can rotate in either direction. As shown in the top half of Figure I.2, the rotational direction of the rotor depends on whether the current is flooding or ebbing. We refer to a turbine employing such a rotor as a bidirectional rotor tidal turbine (BRTT), connoting an axial flow, horizontal axis rotor, bidirectional hydrofoils, fixed pitch, and fixed yaw. A rendering of a commercially developed BRT'T is shown in Figure I.1.

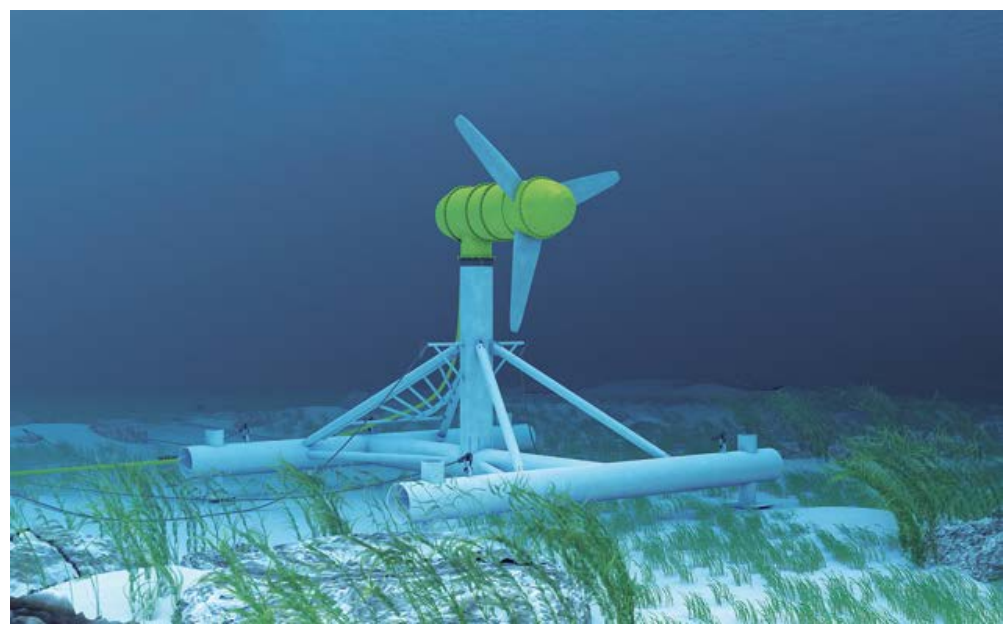

Figure I.1. Rendering of Voith Hydro Ocean Current Technology's BRTT. [2]

There are however, disadvantages to this configuration. Pitch is commonly used to regulate power, maintaining rated power with increasing wind/current speed (Region III) and braking the rotor at cut-out (Region IV). Further, the efficiency of a BRTT rotor is hindered by the use of bidirectional 
hydrofoils, which are typically bi-symmetric (i.e., symmetric about both the chordline and a perpendicular axis at mid-chord) and less hydrodynamically effective than optimized, cambered foils with relatively sharp trailing edges. A study comparing a BRTT to a conventional configuration with variable pitch and yaw found the latter to perform up to $10 \%$ better, producing $4 \%-7 \%$ more energy over its lifetime when availability losses were factored in [1]. However, with reduced O\&M requirements, the BRT'T was found to be more economically effective with a $7.8 \%$ and $9.6 \%$ lower levelized cost of energy assuming 10 year and 15 year lifetimes, respectively.

Some of the performance shortfall of the BRTT can be recaptured with active flow control. Active flow control typically employs some sort of actively controlled device on rotor blades to manipulate aero/hydro-dynamic forces and loads. A wide variety of active flow control mechanisms have been studied, including flaps, microflaps, spoilers, deployable vortex generators, and blowing/suction. Microtabs, shown in Figure I.2, are small (heights of 1\%-2\% of chord) tabs that extend approximately perpendicularly from an air/hydro-foil surface. Applied to a foil trailing edge, they have an effect similar to gurney flaps, pulling the aft stagnation point forward. This effectively changes the airfoil camber and, consequently, global aero/hydro-dynamic forces. As shown illustratively in Figure I.2, deploying and retracting microtabs at different locations on a foil surface can increase rotor performance while maintaining bidirectionality.

In this project, we studied bidirectional hydrofoils for BRTT blades and the use of microtabs to improve rotor performance. 

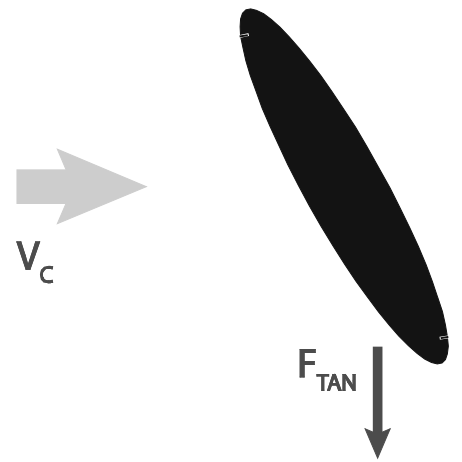

$\uparrow \mathrm{F}_{\mathrm{TAN}}$
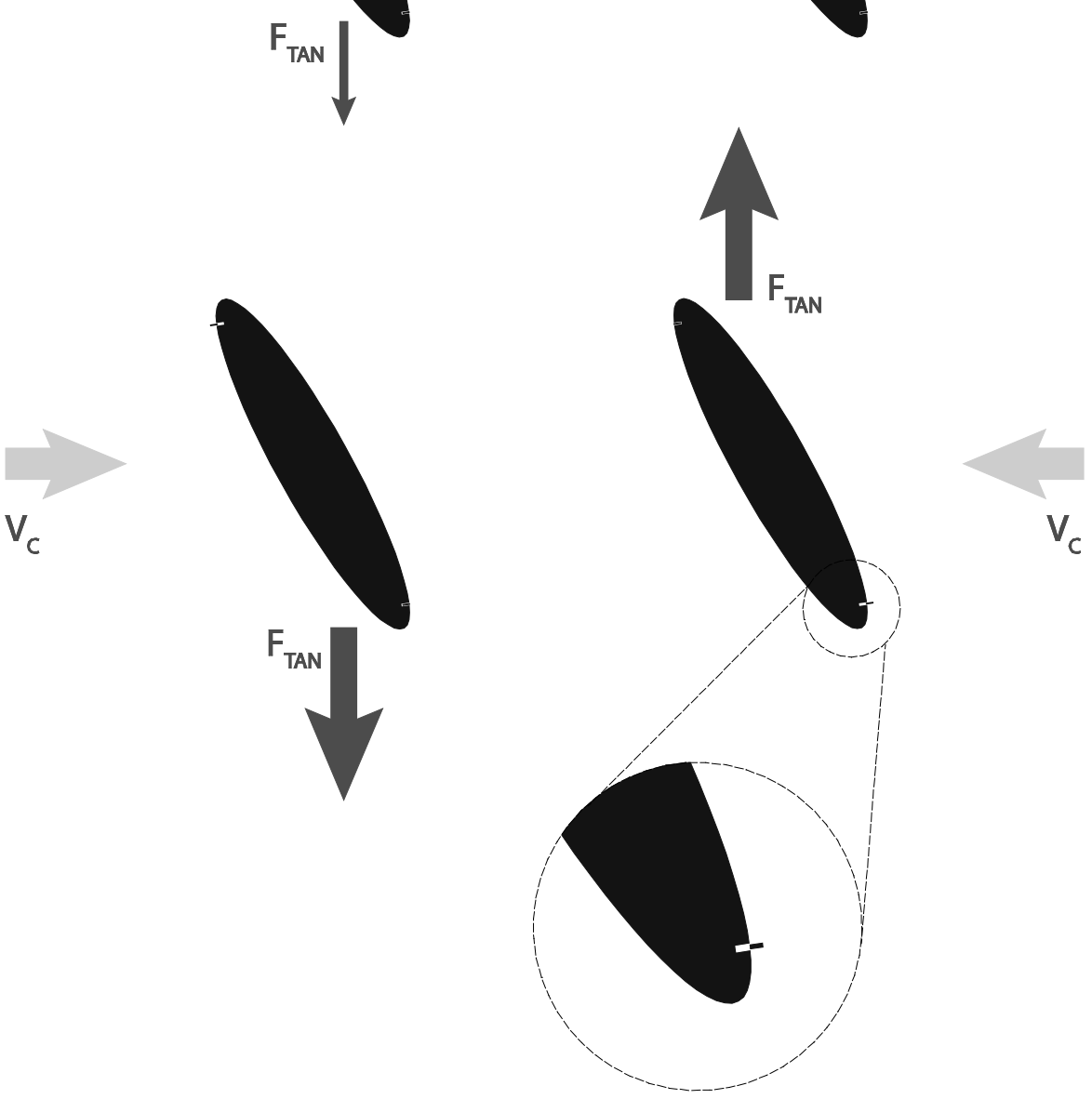

Figure I.2. Blade cross-section of a BRTT with microtabs. $\mathrm{V}_{\mathrm{c}}$ is the current velocity. $\mathrm{F}_{\text {TAN }}$ is the tangential component of the hydrodynamic forces produced by the blade; this is the force component that drives the rotor. In the upper-left, the microtabs are retracted. In the upper-right, the microtabs are also retracted, but the current is reversed, driving the rotor in the opposite direction. In the lower pair of images, the microtabs on the high pressure side of the blade are deployed (as detailed in the callout), increasing the tangential force driving the rotor. 


\section{PROJECT ACTIVITIES: HYDROFOIL AND ROTOR DESIGN AND ANALYSIS}

\section{II.1. COMPUTATIONAL TOOLS}

A variety of tools were used in the design and analysis of the hydrofoils and rotors of this study. Many are very well vetted tools, including:

- XFOIL [3] - Coupled viscous-inviscid flow solver with airfoil design routines (both direct manipulation and inverse design). XFOIL was of limited utility because of the novel geometries required for bidirectional foils.

- OVERFLOW [4] - Reynolds-averaged Navier-Stokes (RaNS) computational fluid dynamics (CFD) flow solver. A very capable viscous, compressible solver with numerous turbulence models. This was used extensively for analysis of the foil designs. Runs were performed time-accurate to accurately capture unsteady flow behavior.

- WT_Perf [5] - Rotor analysis code implementing blade element momentum theory.

- AirfoilPrep [6] - 2-D sectional data pre-processor for air/hydro-foil performance data which are inputted into WT_Perf. Provides post-stall extrapolation via the Viterna method and 3-D stall corrections.

These tools were augmented by a large range of in-house tools, including several developed specifically for this study. They include:

- Tools for manipulating airfoil surface geometries - These included routines for generating various bidirectional foils, interpolating coordinates, fairing segments/adding curvature to corners via cubic and Bezier splines, and adding surface-normal microtabs.

- Grid generation tools - These were used in conjunction with Chimera Grid Tools [7] to automate grid generation.

- Pre- and post-processing routines for OVERFLOW and WT_Perf to automate runs including input file generation, job initiation and monitoring, output file parsing, and plotting. These routines largely facilitated the execution of multiple design iterations.

- Betz optimum rotor design code - A fast, robust code for calculating optimized blade chord and twist distributions. It includes corrections for hub and tip losses.

\section{II.2. HYDROFOILS FOR BIDIRECTIONAL TIDAL TURBINES}

A bidirectional rotor requires air/hydro-foils designed specifically to operate in both directions. As originally conceived, the majority of this project was to focus on the study of microtab applications on elliptical or similar foils. Early in the project, we recognized an opportunity to develop a novel set of bidirectional foils with improved performance over ellipse-like foils. These foils, dubbed the yy foils, came to encompass a significant portion of our work and are presented herein.

\section{II.2.a. Elliptical Hydrofoils and Other Initial Hydrofoil Candidates}

A literature survey revealed little prior work on bidirectional foils. Hoerner briefly discussed elliptical airfoils in his classic aerodynamics text Fluid-dynamic Lift [8]. Thompson filed a patent application for a bidirectional MHK turbine using a bisymmetric hydrofoil [9]. His hydrofoil is 
based on the NACA 67 series, with the forward/leading half of the airfoil reflected about a vertical axis at mid-chord.

An ellipse is the most basic bisymmetric foil. Reflecting a known symmetric foil (i.e., symmetric about its chord line), as Thompson did, can produce performance improvements over the ellipse. However, discarding the original foil's trailing edge significantly affects the foil's global aero/hydrodynamics. The original foil's performance therefore offers little indication of the performance of its reflected/bisymmetric derivative.

We analyzed the aerodynamic performance of an ellipse and a reflected/bisymmetric foil. We used a thickness of $18 \%$ chord, which was estimated to provide sufficient bending strength for an outboard rotor section in water. For the reflected/bisymmetric foil, we considered the NACA 6 series for a starting foil. As Thompson found, the NACA 67-xxx airfoils have maximum thickness at the appropriate location for bisymmetric conversion. The resulting foil is referred to as the Mirrored 67-018 or m67-018 from hereon out.

As shown in Figure II.1, the ellipse and m67-018 have similar geometries. However, as shown in the enlargement in Figure II.2, there are discernible differences.

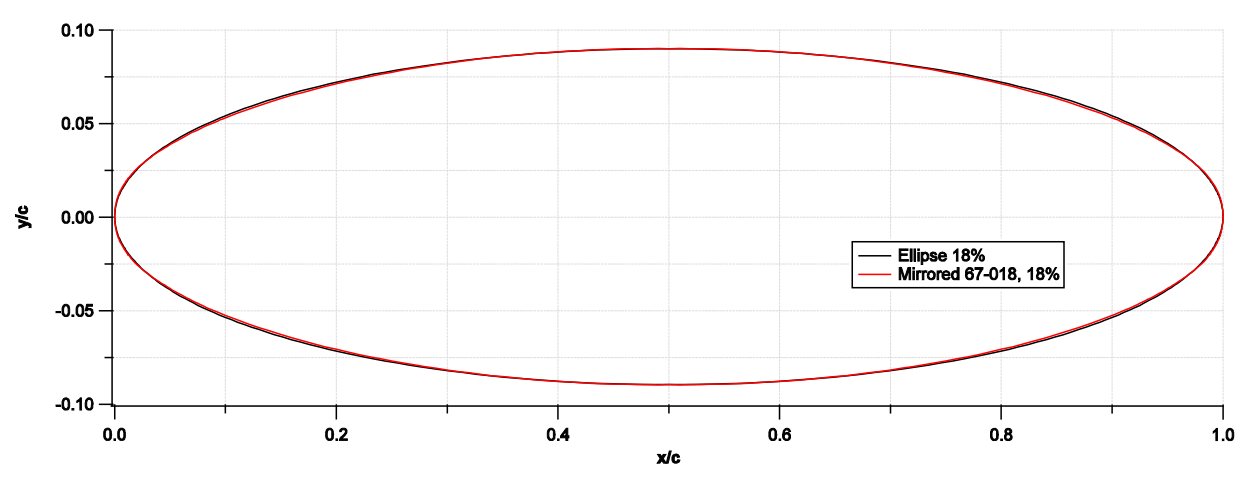

Figure II.1. Ellipse and m67-018 foils, thickness of 18\% of chord.

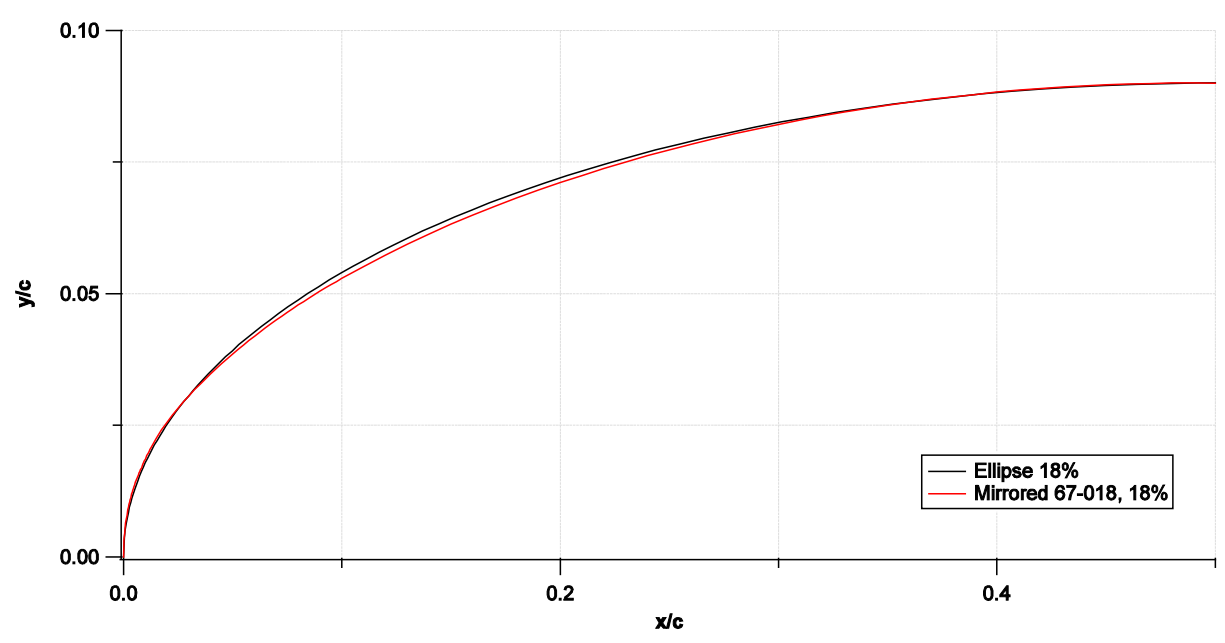

Figure II.2. Enlargement of ellipse and m67-018 foils, thickness of 18\% chord. 
Two yy foils, the yy636-cn-18 and yy636-c03-18, were also designed. The yy foil geometries are not shown, as they are pending patent application (see Section V).

The 18\% ellipse, m67-018, and two yy636 foils were analyzed with RaNS CFD using OVERFLOW. The runs were conducted at $\operatorname{Re}=1.5 \times 10^{6}, \mathrm{M}_{\infty}=0.2$, fully turbulent, over a range of angles of attack. The results are shown in Figure II.3.

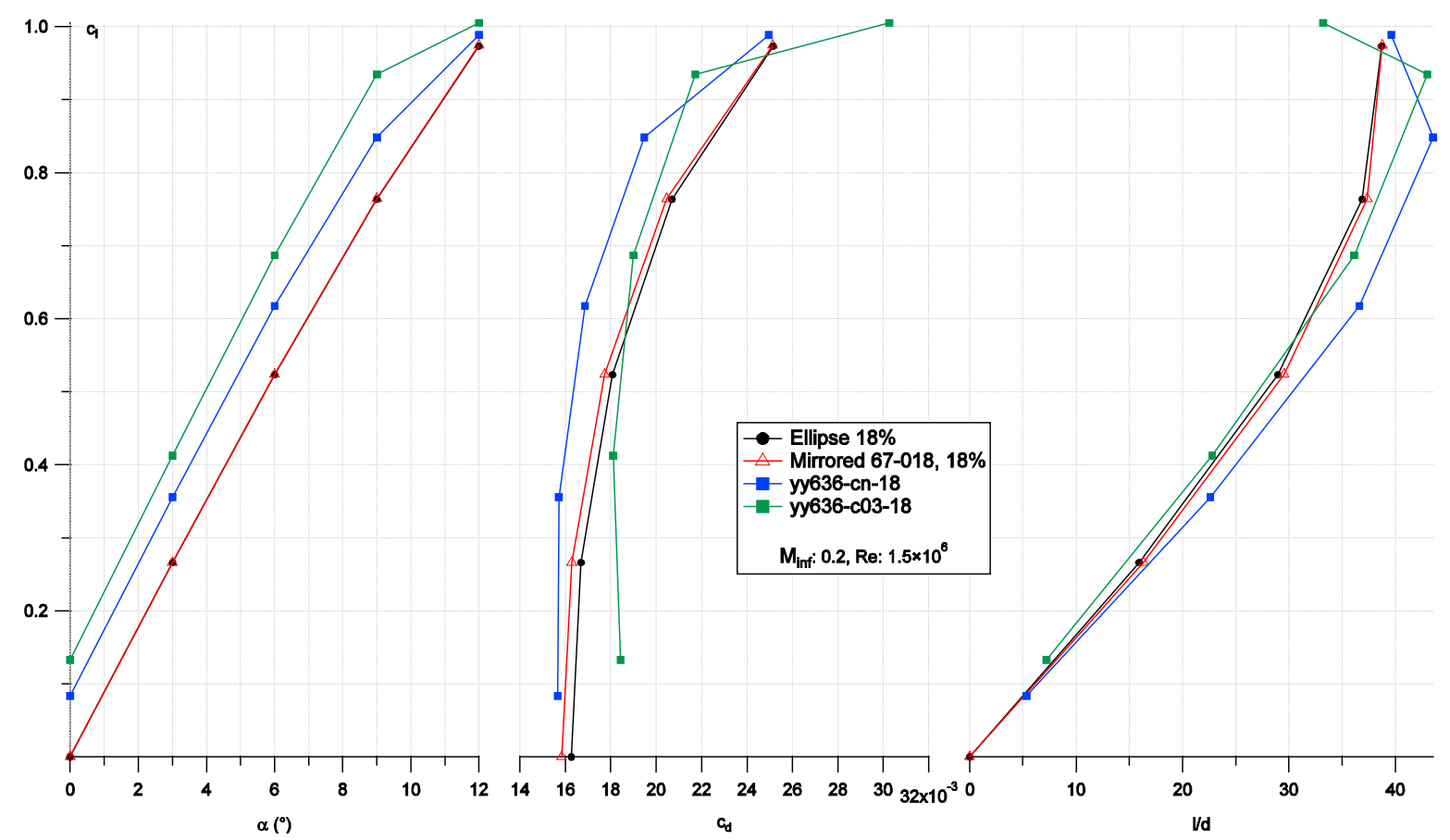

Figure II.3. Comparison of performance of ellipse, m67-018, yy636-cn-18, and yy636-c03-18. $(\mathrm{t} / \mathrm{c})_{\max }=18 \%, \operatorname{Re}=1.5 \times 10^{6}, \mathrm{M}_{\infty}=0.2$, fully turbulent. OVERFLOW.

The ellipse and m67-018 perform very similarly, with the m67-018 slightly better. The yy636 foils both exhibit better lift performance than the ellipse and m67-018. The yy636-cn-18 also has consistently lower drag, with L/D exceeding that of the ellipse and m67018 by as much as $12 \%$ $15 \%$. Based on these findings, the simple ellipse was retained as a baseline and the yy foils were further developed.

\section{II.2.b. yyb07cn Hydrofoils}

After six more design evolutions, the yyb07cn family of foils was reached. This was the final set of yy foils used in subsequent rotor designs. Compared to earlier yy iterations, the yyb $07 \mathrm{cn}$ foils had at least comparable performance in terms of $(1 / d)_{\max }$ (maximum lift-to-drag ratio), but reduced susceptibility to cavitation through purposeful leading edge design to increase $c_{\mathrm{p}, \min }$. Three variants of the yyb07cn were developed: yyb07cn-180, yyb07cn-240, and yyb07cn-350, with (t/c) $\max$ of, respectively, $18 \%, 24 \%$, and $35 \%$. These were analyzed with OVERFLOW at $\operatorname{Re}=1.5 \times 10^{6}, \mathrm{M}_{\infty}=0.2$, fully turbulent. The results are presented in Figure II.4, along with ellipses with corresponding thicknesses. 


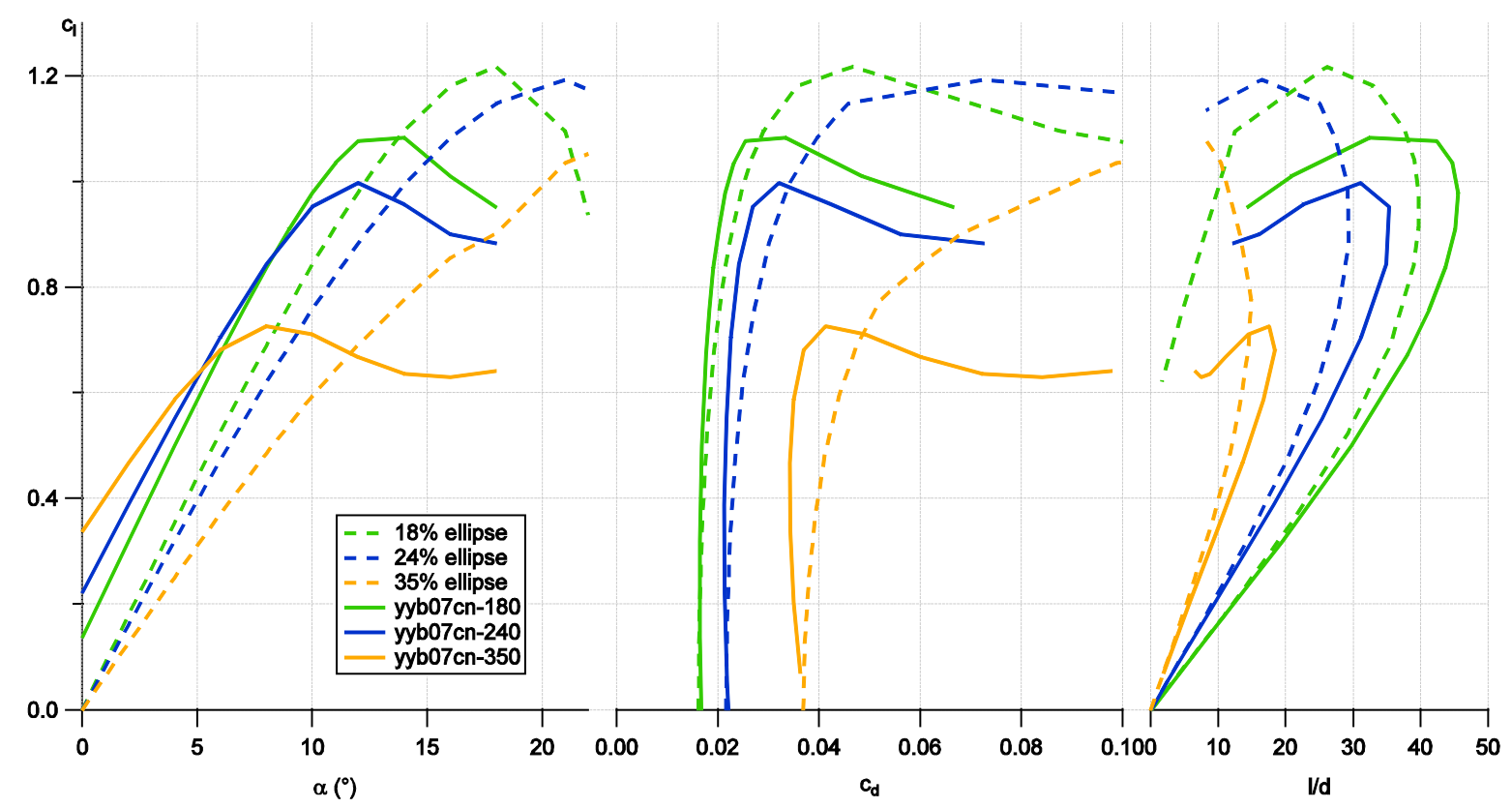

Figure II.4. Hydrodynamic performance of the elliptical and yyb07cn hydrofoil families. Each family has hydrofoils of thickness $18 \% \mathrm{c}, 24 \% \mathrm{c}$, and $35 \% \mathrm{c}$. $\mathrm{Re}=1.5 \times 10^{6}, \mathrm{M}_{\infty}=0.2$, fully turbulent. OVERFLOW.

Compared to the ellipses, the yyb07cn foils exhibit significantly better $(1 / d)_{\max }$. The yyb07-180, yyb07-240, and yyb07-250 have, respectively, 14.7\%, 20.6\%, and $23.7 \%$ higher $(1 / \mathrm{d})_{\max }$ than their corresponding ellipses. As the yyb07cn foils approach stall, the lift curve gradually becomes less linear, curving slightly downward. Upon stall, lift and $\mathrm{l} / \mathrm{d}$ slope downward with increasing angle of attack. Ideally, particularly for stall regulated rotors, the lift curve would remain linear with increasing angle of attack until stall, and then maintain constant lift beyond. It is important to note that in general, 2-D computational analysis of foils is least accurate at and around stall. Characterization in this regime is ideally verified with wind or water tunnel experiments.

\section{II.2.c. Microtabs on Ellipses and yyb07cn Hydrofoils}

Microtabs were first studied on the $18 \%$ ellipse. They were applied to the high pressure surface near the trailing edge at a range of locations: $94 \% \mathrm{c}, 96 \% \mathrm{c}$, and $98 \% \mathrm{c}$. The microtab height was $1 \% \mathrm{c}$ and thickness was $0.2 \%$ c. 


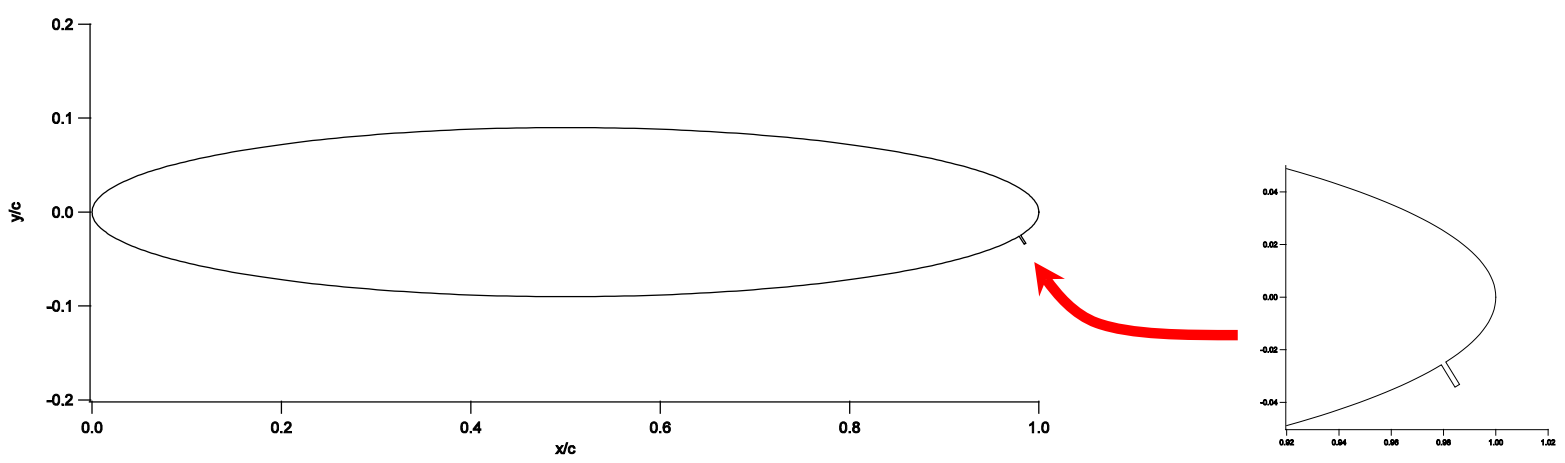

Figure II.5. 18\% ellipse with a 1\%c microtab at $98 \%$ c.

Unlike prior work, the microtabs extended normal to the foil surface instead of the chord line. For computational study, the surface grid of a surface-normal tab requires more effort to generate.

However, it may inject into the flow more effectively, especially given the high curvature at the ends of a bidirectional foil. Surface-normal tabs also have the additional benefit of retracting into a larger volume; internal volume for retraction (and various microtab subsystems) is a particularly important consideration for conventional trailing edges where space is often tightly constrained.

Analysis was performed with OVERFLOW at $\mathrm{Re}=1.5 \times 10^{6}, \mathrm{M}_{\infty}=0.2$, with fully turbulent flow. The results are shown in Figure II.6. This analysis was done in parallel with yy hydrofoil design development, so an older yy hydrofoil, the yy636-cn-18, is included in the figure for comparison.

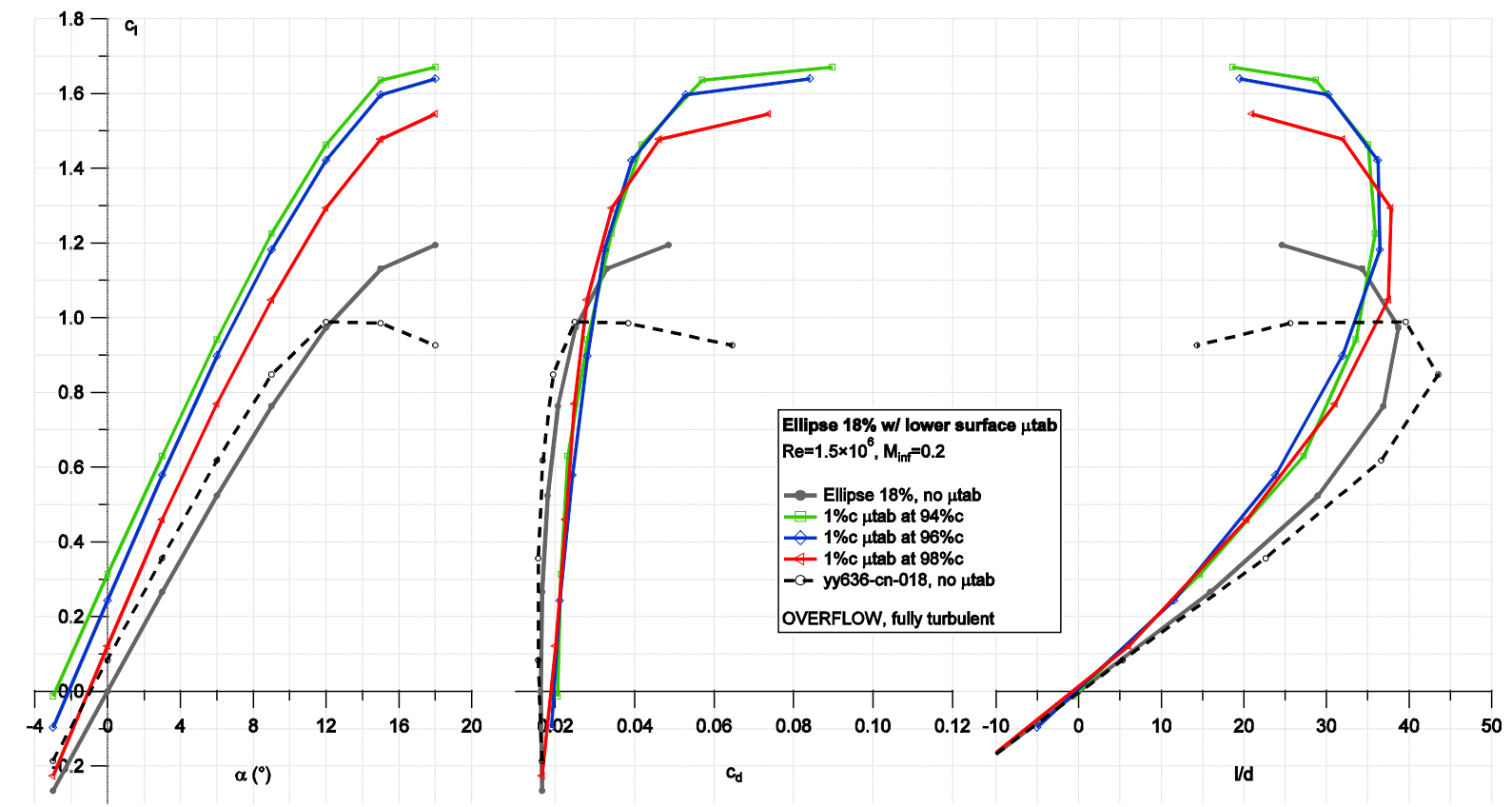

Figure II.6. Performance of the $18 \%$ ellipse with microtab ( $\mu$ tab) on the high pressure surface near the trailing edge. Microtab height: $1 \%$ c. Microtab thickness: $0.2 \%$ c. Re $=1.5 \times 10^{6}$, $\mathrm{M}_{\infty}=0.2$. OVERFLOW. 
The microtabs had a pronounced aerodynamic effect. Most significantly $(\mathrm{l} / \mathrm{d})_{\max }$ decreased with the application of microtabs. Lift was significantly increased, but this was offset by relatively larger increases in drag.

With microtabs, the lift curve shifted up and the lift curve slope slightly increased. This behavior differs somewhat from that of conventional airfoils with microtabs, where the lift curve slope is expected to remain unchanged. On the conventional foil with a microtab near the trailing edge, the aft stagnation point is pulled toward the microtab. In terms of thin airfoil theory, this effectively increases the airfoil camber, offsetting the lift curve upward with a high pressure surface microtab and downward for a suction surface microtab.

The bidirectional foil, however, has a relatively bluff "trailing edge" with high curvature (since the trailing edge must become the leading edge when operating in reverse). It is then likely that flow is separated off the trailing edge as shown with the $18 \%$ ellipse in Figure II.7. In Figure II.7b, the microtab induced the wake downward, effecting a lift increase. However, the separated zone (primarily visible in blue) was not only redirected, but overall reshaped. The separation zone became larger, significantly increasing drag.
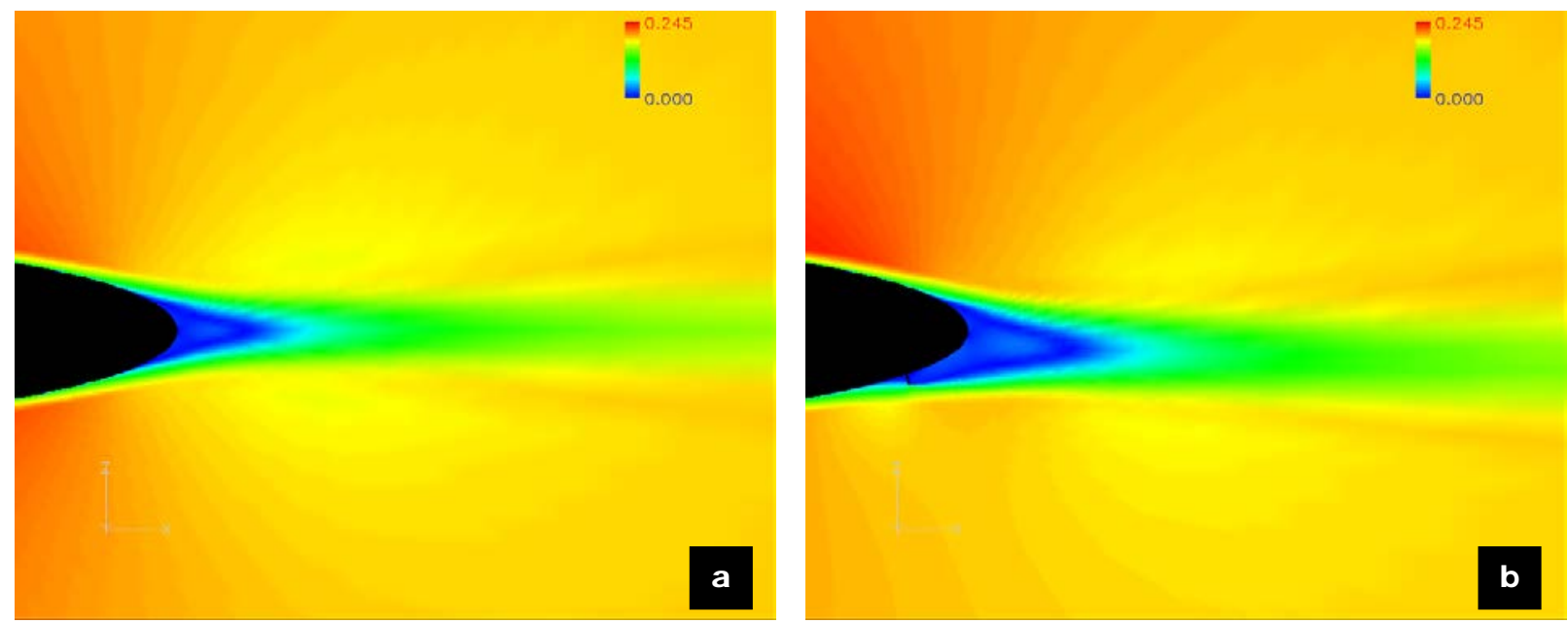

Figure II.7. Velocity magnitude of the $18 \%$ ellipse at $\alpha=0^{\circ}, \mathrm{Re}=1.5 \times 10^{6}, \mathrm{M}_{\infty}=0.2$, fully turbulent. OVERFLOW. a. No microtab. b. Microtab at $94 \%$ c with $1 \%$ c height.

Additionally, the $18 \%$ ellipse exhibited a greater tendency to vortex shed with a microtab, as shown in Figure II. 8 at $\alpha=12^{\circ}$. Vortex shedding can have numerous deleterious effects, including: oscillation of aero/hydro-dynamic forces and, subsequently, structural loads; increased potential for singing in water applications (although this is mitigated by the substantial trailing edge thickness); downstream impacts on other turbines when operating in an array; and downstream environmental impacts (noise, scouring). It may be possible to attenuate the vortex shedding by shaping the tip of the microtab. 

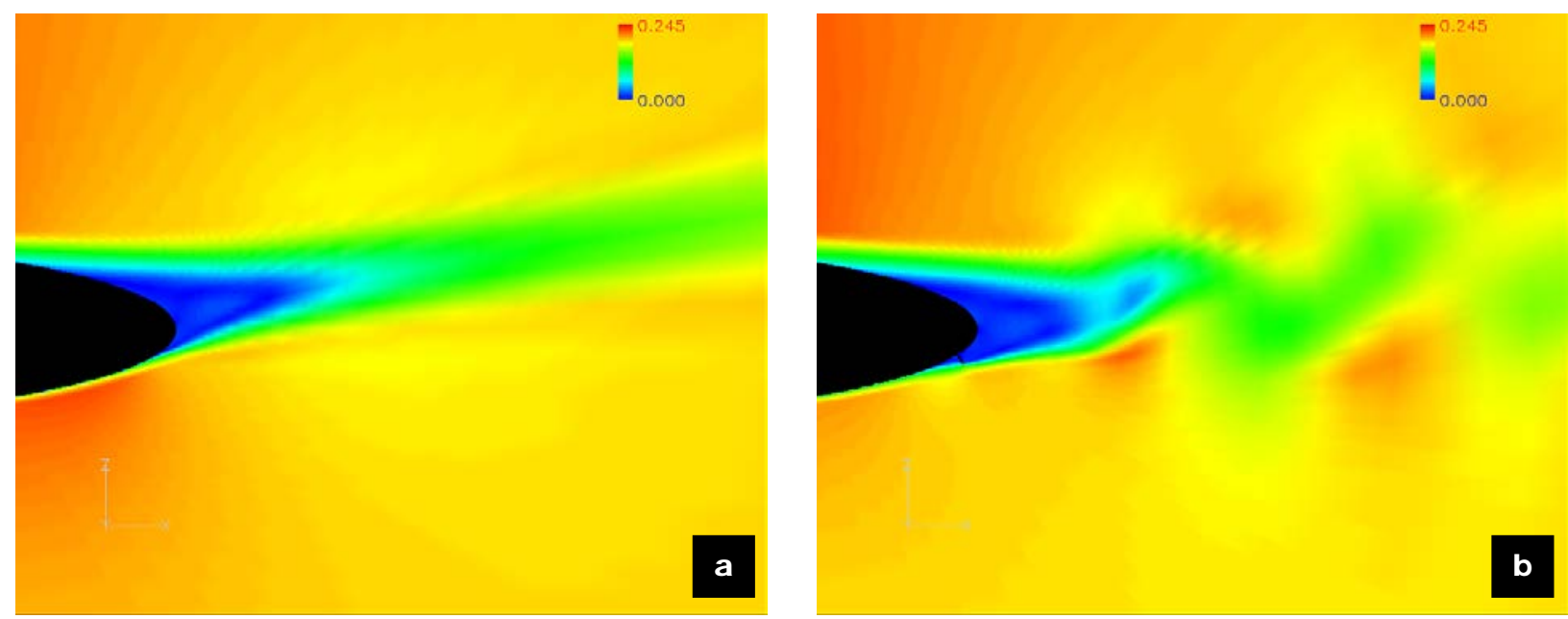

Figure II.8. Velocity magnitude of the $18 \%$ ellipse at $\alpha=12^{\circ}, \mathrm{Re}=1.5 \times 10^{6}, \mathrm{M}_{\infty}=0.2$, fully turbulent. OVERFLOW. a. No microtab. b. Microtab at $98 \%$ c with $1 \%$ c height; note vortex shedding.

Analysis of microtabs on yy hydrofoils yielded very different results, as shown on the yyb07cn-180 in Figure II.9 (Figure II.4 shows the no microtab case). As on the ellipse, a microtab was applied on the high pressure surface with a height of $1 \% \mathrm{c}$, over a range of locations near the trailing edge. As with the ellipse, lift increased; the curves shifted upward with more forward tab location. Interestingly, the lift curve slope not only slightly increased, but it straightened; i.e., the linear portion was extended to higher angles of attack. Unlike the ellipse, drag did not significantly increase. As a result, hydrodynamic performance as indicated by $(1 / d)_{\max }$ improved with the microtab on the yyb07cn-180.

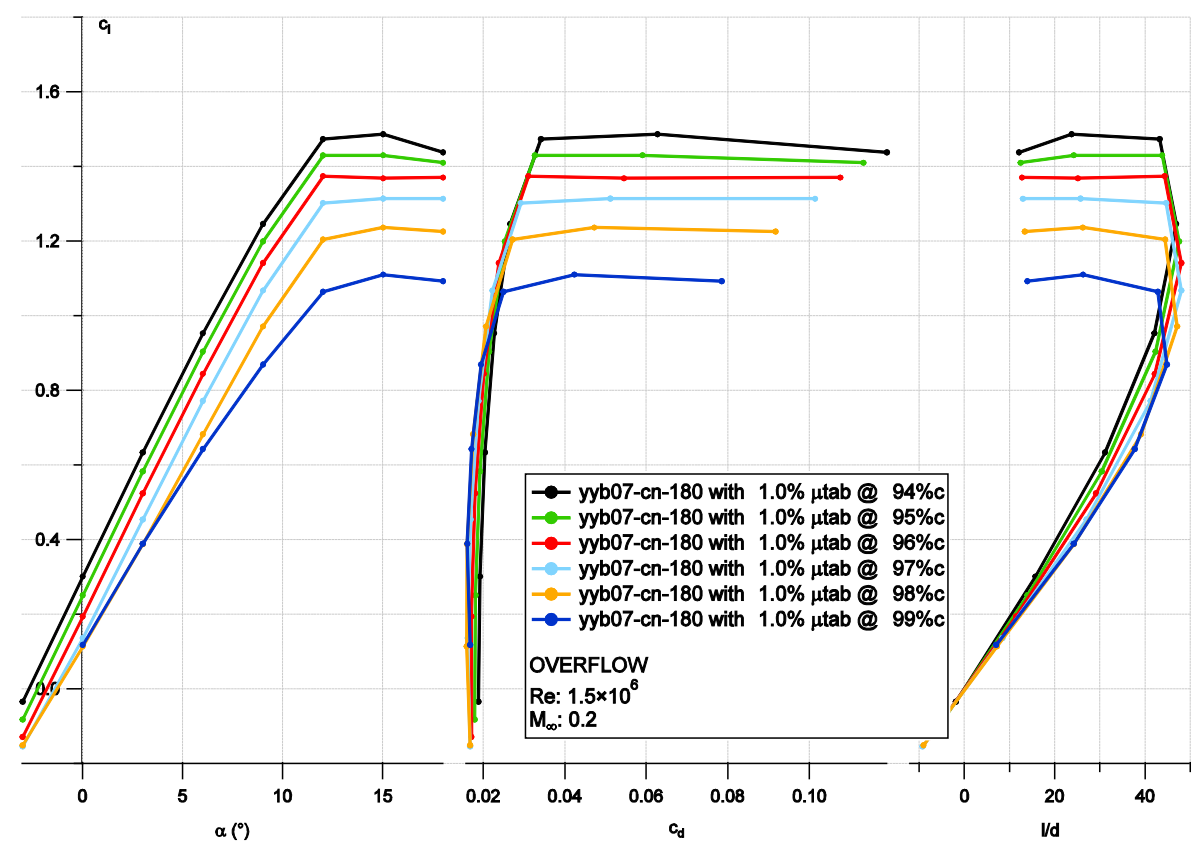

Figure II.9. Hydrodynamic performance of the yyb07cn-180 with a microtab on the high pressure surface. Microtab height: $1.0 \%$ c. Microtab location: $94 \% \mathrm{c}-99 \%$ c. OVERFLOW, Re $=1.5 \times 10^{6}, \mathrm{M}_{\infty}=0.2$, fully turbulent. 
Contrary to the findings on the ellipse, $(1 / \mathrm{d})_{\max }$ improved on all of the yy foils on which microtabs were applied. With conventional, streamlined trailing edges, aft microtabs can be equated to changes in camber, as with gurney flaps or plain flaps. With a bluff trailing edge, the effect is less straightforward. Microtabs can be thought of as a mechanism for reshaping the trailing edge separation zone. As shown with the ellipses and yy foils, this can have widely different impacts on aero/hydro-dynamic performance, underscoring that microtabs are not a simple design "add-on", but need to be thoroughly incorporated into the foil design and selection process.

Microtab height and location were studied on the yyb07cn-180. In Figure II.9 through Figure II.12, microtab heights of $1.0 \% \mathrm{c}, 1.5 \% \mathrm{c}$, and $2.0 \% \mathrm{c}$ were cross-compared with chordwise locations of $94 \%$ c to $99 \%$ c on the high pressure surface. As with prior analyses, the runs were made with OVERFLOW. Some runs - particularly larger microtabs further aft and at higher angles of attack did not successfully converge and are excluded from the plots. However, more than enough runs were successful to distinguish sizing and placement trends.

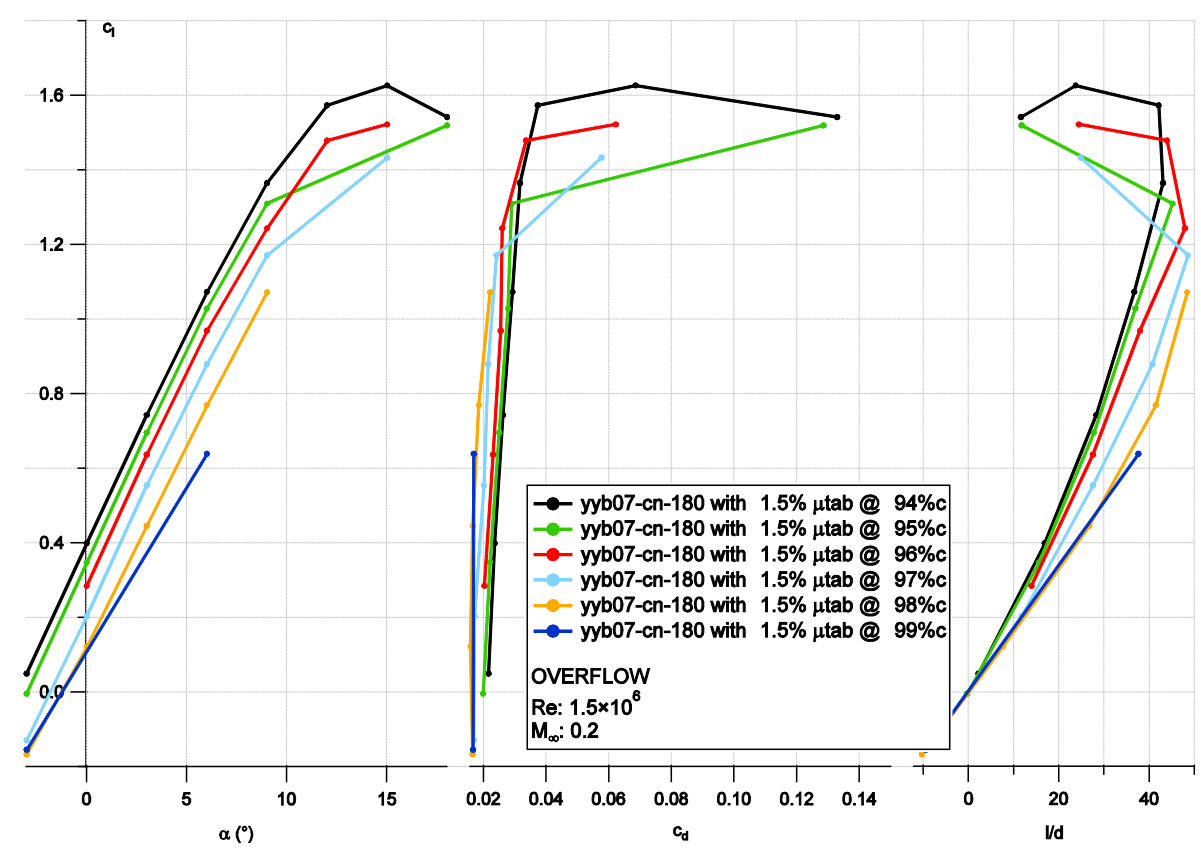

Figure II.10. Hydrodynamic performance of the yyb07cn-180 with a microtab on the high pressure surface. Microtab height: $1.5 \%$ c. Microtab location: $94 \% \mathrm{c}-99 \%$ c. OVERFLOW, $\mathrm{Re}=1.5 \times 10^{6}, \mathrm{M}_{\infty}=0.2$, fully turbulent. 


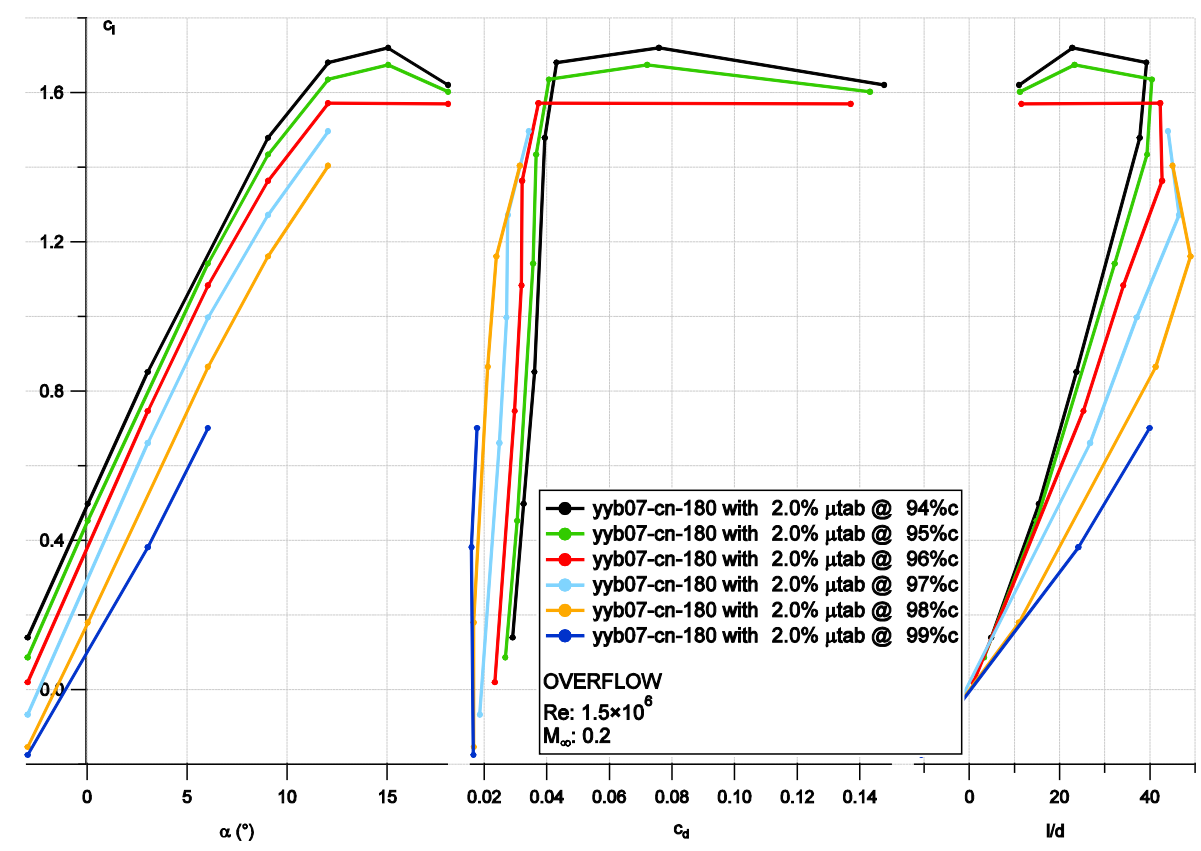

Figure II.11. Hydrodynamic performance of the yyb07cn-180 with a microtab on the high pressure surface. Microtab height: $2.0 \%$ c. Microtab location: $94 \% \mathrm{c}-99 \%$ c. OVERFLOW, $\mathrm{Re}=1.5 \times 10^{6}, \mathrm{M}_{\infty}=0.2$, fully turbulent.

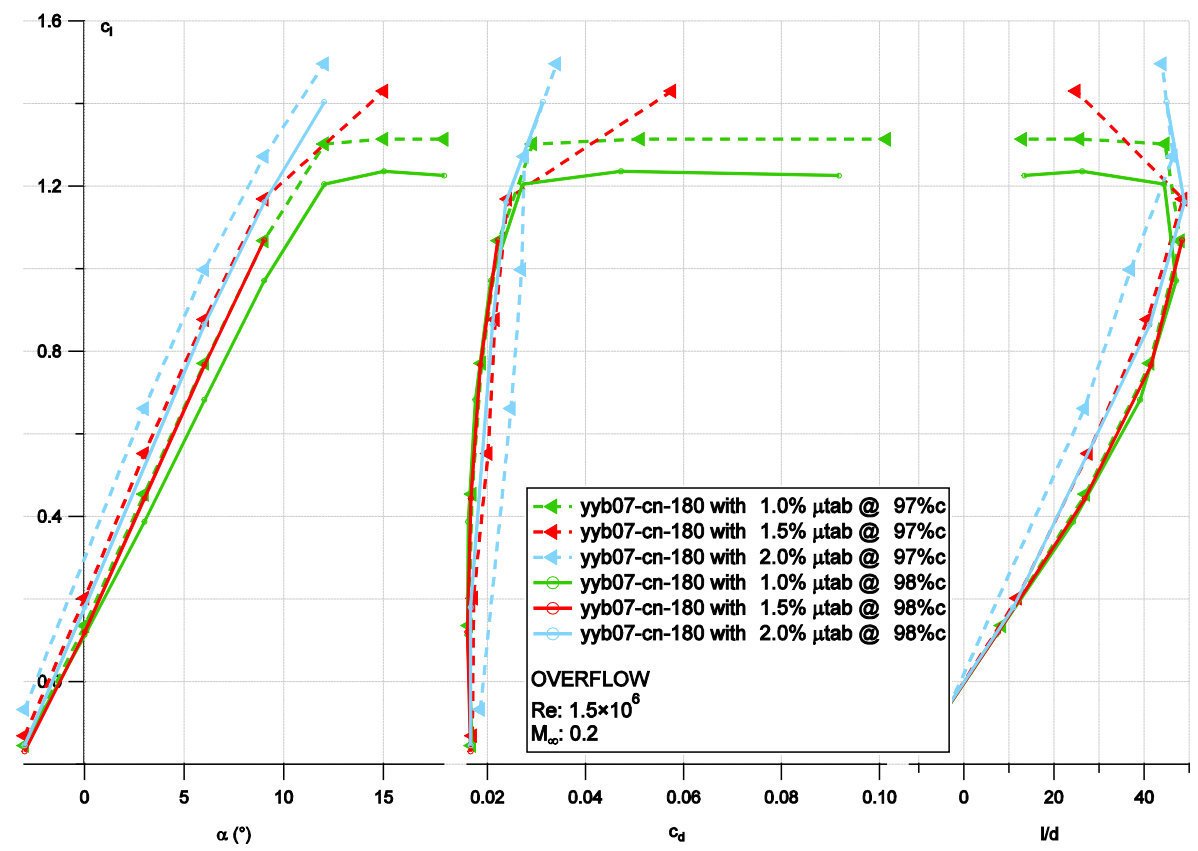

Figure II.12. Hydrodynamic performance of the yyb07cn-180 with a microtab on the high pressure surface. Microtab height: $1.0 \%$ c, $1.5 \%$ c, $2.0 \%$ c. Microtab location: $97 \%$ c, $98 \%$ c. OVERFLOW, $R e=1.5 \times 10^{6}, \mathrm{M}_{\infty}=0.2$, fully turbulent.

As expected, the effect of the microtabs is akin to increasing camber (as with a plain flap), shifting the lift curve upward. Larger and more forward microtab configurations increased lift more. However, the lift increments came with a corresponding drag penalty, affecting the key metric of 
$1 / \mathrm{d}$. With the $1.0 \% \mathrm{c}$ height microtabs, $(1 / \mathrm{d})_{\max }$ was approximately the same across all chordwise locations, but occurred at higher $\mathrm{c}_{1}$ values with more forward locations. With the $1.5 \% \mathrm{c}$ and $2.0 \% \mathrm{c}$ height microtabs, $(\mathrm{l} / \mathrm{d})_{\max }$ decreased as the tab location moved forward. With all microtab heights, $(\mathrm{l} / \mathrm{d})_{\max }$ was highest when the microtabs were at $96 \% \mathrm{c}-98 \% \mathrm{c}$ chordwise location. Figure II.12 compares performance across the range of microtab heights, located at $97 \% \mathrm{c}$ and $98 \% \mathrm{c}$. At the $98 \%$ c location, the $1.0 \% \mathrm{c}$ and $1.5 \%$ height microtabs performed comparably. The $2.0 \% \mathrm{c}$ height microtab has approximately the same $(1 / d)_{\max }$, but lower $1 / d$ at $c_{1}$ values below $(1 / d)_{\max }$. This is significant as the rotor design will likely employ a design $c_{1}$ slightly below $(l / d)_{\max }$ (this provides some margin in gusting winds/surging current). At $97 \% \mathrm{c}$, the $1.0 \% \mathrm{c}$ height microtab outperformed the taller microtabs, with comparable $1 / \mathrm{d}$ to $1.0 \% \mathrm{c}$ and $1.5 \% \mathrm{c}$ microtabs at $98 \% \mathrm{c}$.

Overall, there appears to be little benefit from the taller $1.5 \% \mathrm{c}$ and $2.0 \% \mathrm{c}$ microtabs. The $1.0 \% \mathrm{c}$ microtab at $94 \%$ c to $97 \%$ c had comparable $1 / \mathrm{d}$ performance over a range of $\mathrm{c}_{1, \max }$, providing a range of candidates for the rotor designer. In general, it may be beneficial to select a more forward (i.e., more toward the chordwise center of the hydrofoil) tab location. Recall that for our application with a bidirectional rotor, once the current reverses, the aft, pressure side deployed microtab becomes a forward, suction side retracted microtab. The retracted microtab will be a finite discontinuity on the surface. Moving this further aft of the leading edge may limit disruption of the boundary layer, benefiting general hydrodynamic performance and cavitation inception.

Considering all of the above, the hydrofoils selected for the BRTT blades were the yyb07cn family. The outboard hydrofoil was the yyb07cn-180, with microtabs of $1.0 \%$ c height at $95 \%$ chordwise location.

\section{II.2.d. Blade Design and Analysis}

The Betz optimum code introduced in Section II.1 was used to design three blades:

- e-B02 - using elliptical hydrofoils

- yy-B02 - using yyb07cn hydrofoils

- yy $\mu \mathrm{t}-\mathrm{B} 02$ - using yyb07cn hydrofoils with microtabs in the outboard region

$18 \%$ c thick hydrofoils were used in the outboard region of the blade, from $70 \% \mathrm{R}$ to the tip (where $\mathrm{R}$ is the rotor radius or blade length). $24 \% \mathrm{c}$ thick hydrofoils were used in the mid region from $35 \% \mathrm{R}$ to $70 \% \mathrm{R}$. 35\% thick hydrofoils were used in the inboard region from the root to $35 \% \mathrm{R}$. Based on a prior preliminary MHK rotor design study, blades were designed for a three-bladed rotor, tip speed ratio (TSR) of 4, inflow of $2.0 \mathrm{~m} / \mathrm{s}$, and a rotor radius of $2.5 \mathrm{~m}$.

The blades were analyzed on three-bladed rotors with blade element momentum (BEM) theory via WT_Perf. The two dimensional hydrofoil data from OVERFLOW was extrapolated to post-stall with the Viterna method implemented in NREL's AirfoilPrep. Note that 3-D stall corrections were not applied, as they did not return reasonable values; this may be a consequence of the unconventional hydrofoil geometries. The performance of the blades is presented in Figure II.13. 


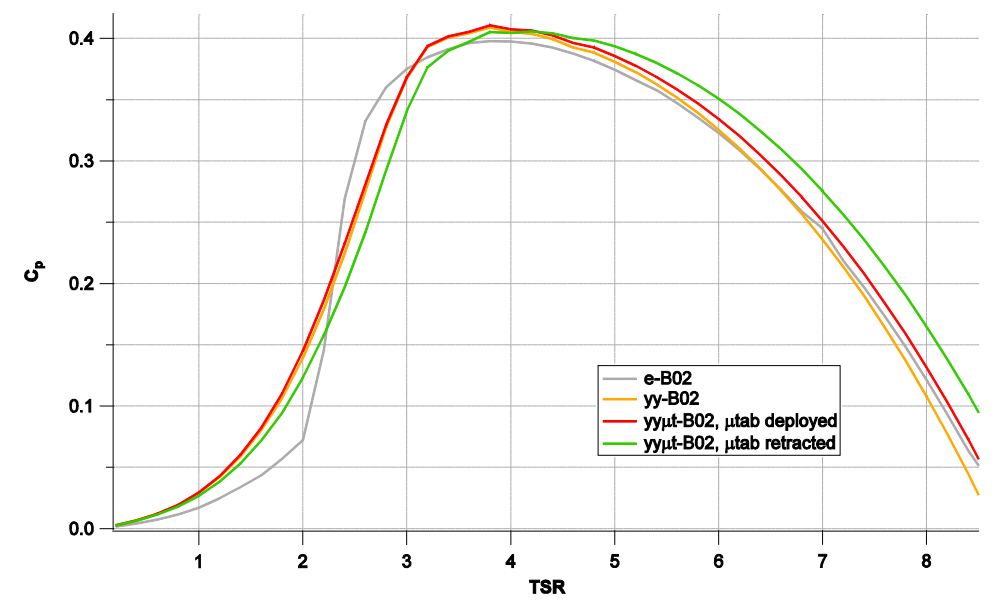

Figure II.13. $\mathrm{C}_{\mathrm{P}}-\mathrm{TSR}$ curves of three-bladed rotors using the e-B02 (using elliptical hydrofoils), yy-B02 (using yyb07cn hydrofoils), yy $\mu \mathrm{t}-\mathrm{B} 02$ (using yyb07cn hydrofoils and designed for outboard microtabs; results are shown with microtabs deployed and retracted) blades.

The yy-B02 rotor exhibits better performance than the e-B02 rotor except a limited range of TSRs between 2.3 and 3.1. The addition of microtabs on the yy $\mu$ t-B02 rotor (with microtabs at outboard locations) slightly improves $C_{P}$ at TSRs less than 4 . Above TSR of 4 , the improvement is more significant. The yy $\mu \mathrm{t}-\mathrm{B} 02$ with retracted microtabs has the same blade chord and twist distribution as the yy $\mu \mathrm{t}-\mathrm{B} 02$, but no microtabs were applied in the outboard region. Retracting the microtabs primarily shifts the curve to the right (toward higher TSRs). Below TSRs of 4, this degrades performance as expected. Interestingly, above TSR of 4, $C_{P}$ is higher with the microtabs retracted. The blades were applied to a variety of rotor configurations and are presented and discussed in the next section. 


\section{ENERGY AND ECONOMIC ANALYSIS}

To assess the economic viability of rotors using the yy-B02 and yy $\mu \mathrm{t}-\mathrm{B} 02$ blades, we performed a series of energy production calculations. While energy production is only one part of cost analysis, at this stage of design, it alone can serve as a useful metric of relative economic viability between different configurations.

\section{III.1. CHARACTERISTIC CURRENT SPEEDS}

Gooch et al measured current speeds at a number of prospective tidal power sites in the Pacific Northwest [10]. Current speed probability distributions of five of these sites are shown in Figure III.1. The overall mean current speed of these five sites is $0.87 \mathrm{~m} / \mathrm{s}$, with maxima ranging from 1.9 $\mathrm{m} / \mathrm{s}$ to $3.3 \mathrm{~m} / \mathrm{s}$. Figure III.1 also includes the mean speed distribution across the five sites and a Rayleigh-based distribution with an equal mean, $0.87 \mathrm{~m} / \mathrm{s}$. The Rayleigh distribution follows the mean speed distribution well and, as this is a speculative study unassociated with any particular sites, was used in all of the following energy production analyses.

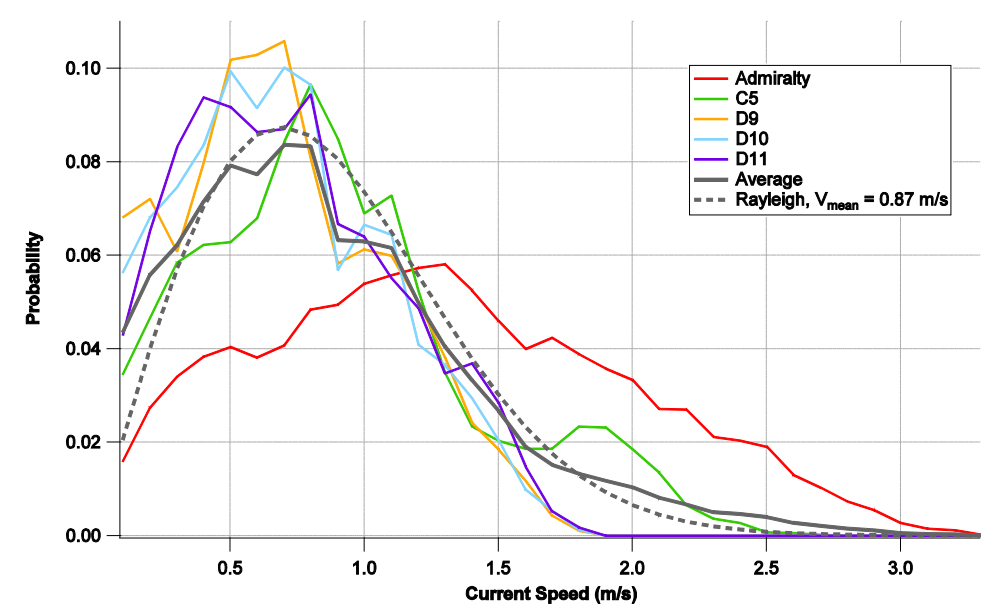

Figure III.1. Current speed probability distributions of five prospective tidal power sites. A Rayleigh-based distribution with an equal mean of $0.87 \mathrm{~m} / \mathrm{s}$ is included.

\section{III.2. TURBINE PARAMETERS}

Based on a preliminary analysis, a three-bladed rotor with a diameter of $38 \mathrm{~m}$ and a rated power of $60 \mathrm{~kW}$ were selected. This yielded capacity factors around 30\%. Compared to wind turbines, this rotor is large relative to its power rating. This is primarily due to the low current speeds, with a mean of $0.87 \mathrm{~m} / \mathrm{s}$. Actual installations would favor faster, more economical sites. Gooch et al [10], for example, measured a site with a mean current speed of $1.95 \mathrm{~m} / \mathrm{s}$. This rotor configuration remains useful for economic analysis at this stage of design where we focus on relative energy production.

\section{III.3. FULL VARIABLE SPEED OPERATION}

Modern wind turbines employ variable speed generators to allow their rotors to operate continuously at or near $C_{p, \max }\left(\right.$ and, hence, $\lambda_{\text {opt }}$ ) as wind speed varies. The rotors run at $C_{p, \max }$ through much of Region II, deviating when generator speed limits, maximum tip speed, or rated power is reached. 
Variable speed rotors were designed with the e-B02, yy-B02, and yy $\mu$ t-B02 blades, denoted by the suffix -Rvs (e.g., yy $\mu \mathrm{t}-\mathrm{B} 02-\mathrm{R} v s)$. The operating tip speed ratios and blade incidence angles of each rotor were optimized for maximum $C_{p}$. The resulting TSRs and blade incidence angles varied between rotors, with values near (but not exactly) 4 and $0^{\circ}$, respectively. Maximum power production was limited to the rated power, $60 \mathrm{~kW}$, and cut-out was designated at $3 \mathrm{~m} / \mathrm{s}$. No tip speed constraint was applied. The resulting power curves are shown in Figure III.2.
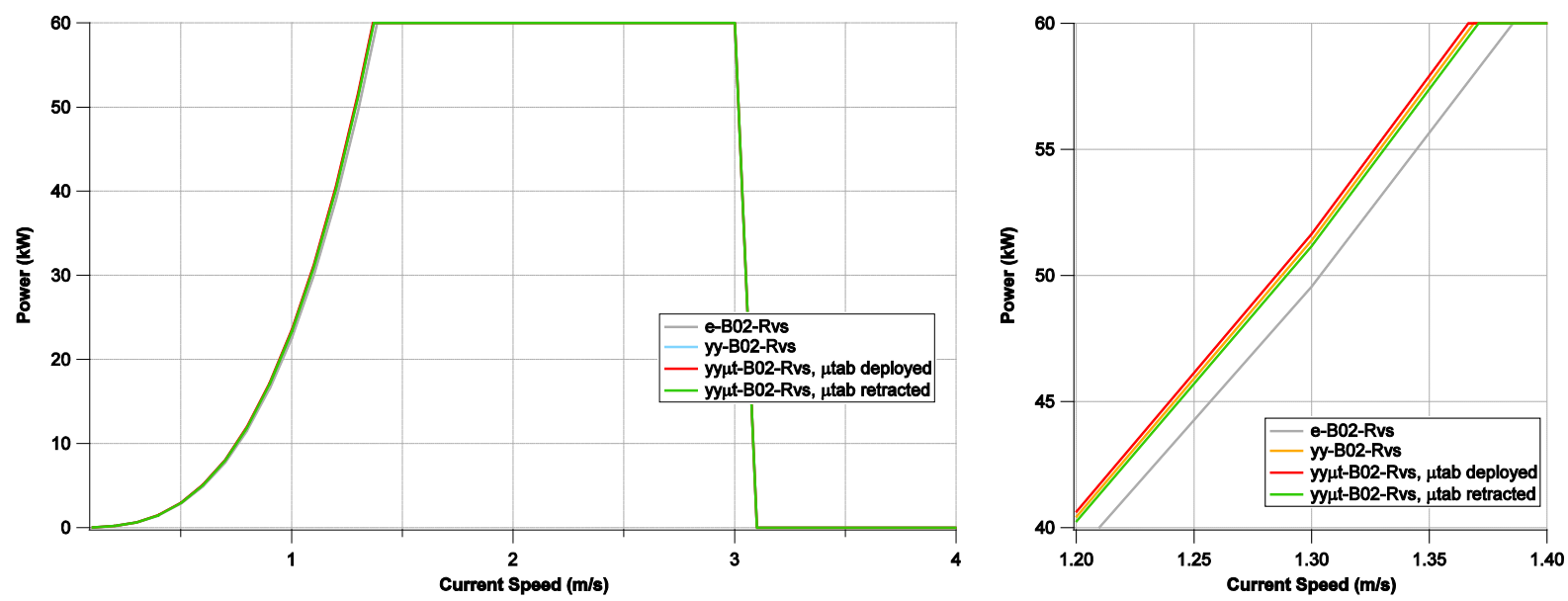

Figure III.2. Power curves of variable speed rotors with elliptical hydrofoils (e-B02-R vs), yy hydrofoils (yy-B02-R $v s$ ), yy hydrofoils with microtabs deployed (yy $\mu t-B 02-R v s)$, and yy hydrofoils with microtabs retracted (yy $\mu t-B 02-\mathrm{R} v s)$. In the left plot (a), the curves appear to almost overlap. In the right plot (b), a zoom-in to Region II shows the performance differences between the rotors.

In Figure III.2a (left), the green curve appears dominant because it largely overlaps the other power curves. However, the detail in Figure III.2b shows that the rotors with the yyb07cn hydrofoils reached rated power more quickly (that is, at lower current speeds) than the rotor with elliptical hydrofoils. Furthermore, yy $\mu \mathrm{t}-\mathrm{B} 02-\mathrm{R} v s$ with deployed microtabs achieved rated power before the yy-B02-R $v$ (no microtabs) and yy $\mu \mathrm{t}-\mathrm{B} 02-\mathrm{R} v s$ with retracted microtabs. The effects on energy capture are tabulated in Table III-1.

Table III-1. Capacity factors of variable speed rotors (-B02-R $v$ s) assuming power regulation to rated power, cut-out at $3 \mathrm{~m} / \mathrm{s}$, and a Rayleigh distribution with a mean speed of $0.87 \mathrm{~m} / \mathrm{s}$.

\begin{tabular}{|l|l|c|}
\hline Rotor & $\mathbf{C}_{\mathbf{P}, \max }$ & Capacity Factor (\%) \\
\hline e-B02-Rvs & 0.399 & 34.9 \\
\hline yy-B02-Rvs & 0.414 & 35.7 \\
\hline yy $\mu$ t-B02-Rvs, $\mu$ tab deployed & 0.416 & 35.8 \\
\hline yy $\mu$ t-B02-Rvs, $\mu$ tab retracted & 0.412 & 35.6 \\
\hline
\end{tabular}


The yy $\mu \mathrm{t}-\mathrm{B} 02-\mathrm{R} v$ s rotor with deployed microtabs has the highest energy capture of the rotor configurations, with a capacity factor $0.25 \%$ (0.1 percentage points) higher than the yy-B02-R $v$ s rotor and 2.6\% (0.9 percentage points) higher than the rotor with elliptical hydrofoils. As expected, with microtabs retracted, the energy capture falls below that of the yy-B02-R $v$ s, but notably, remains significantly higher than that of the e-B02-R $v$ s.

Power regulation of variable speed wind turbines is generally effected through variable blade pitch. In our BRTT, we expect to employ fixed pitch (as well as fixed yaw), a key design deviation from modern wind turbines with the intended benefit of decreased O\&M costs. An alternative power regulation mechanism (e.g., active hydrodynamic devices such as microtabs applied in other configurations, electrodynamic devices, etc.) is therefore required to realize the variable speed power curves and energy production presented above. As this may not be feasible, alternate operation and configurations are considered below.

\section{III.4. CONSTANT SPEED OPERATION WITH STALL REGULATION}

Early wind turbines employed simple constant speed generators and aerodynamic stall for power regulation. Constant speed generators are less efficient in Region II because TSR varies with wind speed, precluding extended operation at $\lambda_{\text {opt }}$; however, capital and O\&M costs are lower. Power regulation via stall obviates the need for an active regulation mechanism (e.g., pitch), again lowering capital and O\&M costs. However, in general, it is more difficult to main optimum energy capture in Region III with stall regulation.

To explore constant speed operation with stall regulation, a series of rotors were designed with the e-B02, yy-B02, and yy $\mu$ t-B02 blades, with various rotor speeds and blade incidence angles to optimize energy capture. These are designated by an -Rcs tuple.

In some cases, cut-out was specified (e.g., at rated power). Upon cut-out, a rotor could be stopped without active pitch via a mechanism such as an electrodynamic brake. An alternate method could be available to tidal turbines. At slack, if the current speed is expected to exceed the cut-out speed in the next tidal cycle, the rotor could be preemptively parked. This is feasible because of the high degree of predictability of tides, both over the long term from harmonic models and in the short term from existing, widely deployed instrumentation. The turbine would miss energy capture over most of the cycle (but not necessarily all; it could be unparked as current speeds fall toward slack). However, higher speeds are encountered less frequently, so as long as the cut-out speed is sufficiently high, the overall impact on energy capture would be low.

Figure III.3 shows the power curve of rotor e-B02-Rcs,opt which uses elliptical hydrofoils. It exhibits behavior common to many stall regulated rotors. The power curve first climbs to rated power at approximately $1.6 \mathrm{~m} / \mathrm{s}$, then falls off sharply as sections of the blades stall. As current speed continues to increase, power production begins to increase again as the blade enters a very high angle of attack, post-stall regime. Figure III.3 includes two variations of the power curve. In the solid curve, the turbine is prescribed to cut-out before exceeding rated power. Note that this occurs at approximately $3.4 \mathrm{~m} / \mathrm{s}$, which is significantly faster than any speeds occurring in the distributions in Figure III.1. The dashed line shows how power would continue to increase without cut-out.

Preferably, power output would remain constant upon reaching rated power. There is significant power and energy loss in the dip in the power curve between $1.6 \mathrm{~m} / \mathrm{s}$ and $3.4 \mathrm{~m} / \mathrm{s}$. However, a flat rooftop in this region requires purposeful tailoring of air/hydro-foils to achieve and, unsurprisingly, is not exhibited by simple ellipses. 


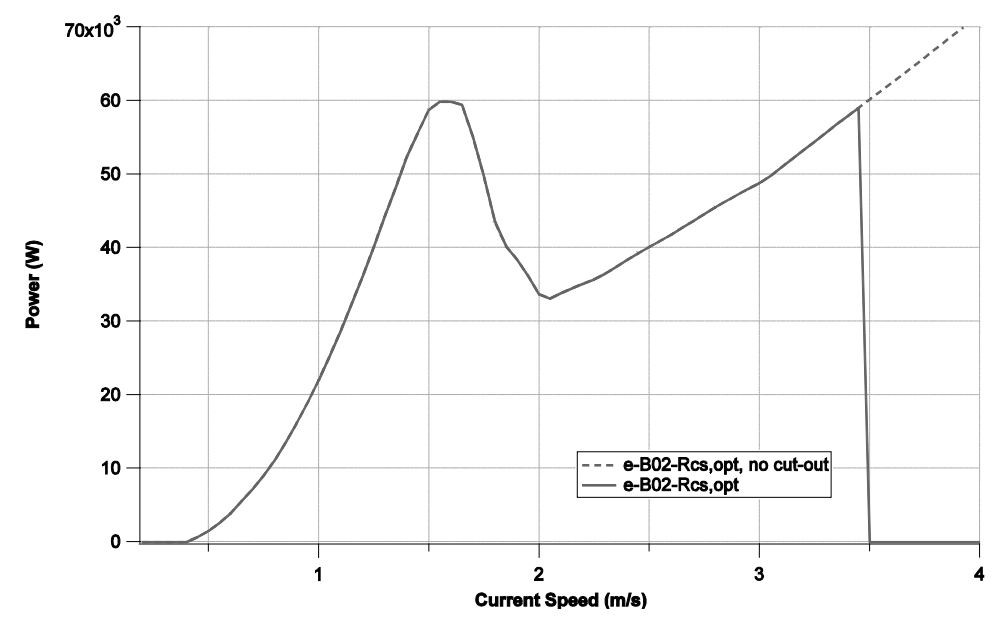

Figure III.3. Power curve of e-B02-Rcs,opt, a stall-regulated rotor with a rated power of $60 \mathrm{~kW}$. The dashed line represents power production without a prescribed cut-out.

Applying the Rayleigh distribution with a mean speed of $0.87 \mathrm{~m} / \mathrm{s}$, the capacity factor of the e-B02$\mathrm{R} c s, o p t$ rotor is $34.9 \%$. This is compared with other rotors (presented below) in Table III-2.

Table III-2. Capacity factors of constant speed rotors (-B02-Rcs) assuming prescribed cutout to prevent exceeding rated power and a Rayleigh distribution with a mean speed of $0.87 \mathrm{~m} / \mathrm{s}$.

\begin{tabular}{|l|l|c|}
\hline Rotor & Description & $\begin{array}{c}\text { Capacity } \\
\text { Factor (\%) }\end{array}$ \\
\hline e-B02-Rcs,opt & Elliptical hydrofoils & 31.2 \\
\hline yy-B02-Rcs,opt & yyb07cn hydrofoils & 31.3 \\
\hline yy-B02-Rcs,stall & yyb07cn hydrofoils, stall regulated & 25.9 \\
\hline yy $\mu$ t-B02-Rcs,opt-dep & $\begin{array}{l}\text { yyb07cn hydrofoils with microtabs, optimized } \\
\text { for energy capture with microtabs deployed }\end{array}$ & 31.5 \\
\hline yy $\mu$ t-B02-Rcs,fail & $\begin{array}{l}\text { yyb07cn hydrofoils (no microtabs), optimized } \\
\text { for energy capture with microtabs deployed; } \\
\text { this represents a failure condition with the } \\
\text { microtabs stuck retracted }\end{array}$ & 29.9 \\
\hline yy $\mu$ t-B02-Rcs,opt & $\begin{array}{l}\text { yyb07cn hydrofoils with microtabs, optimized } \\
\text { for energy capture with microtabs optimally } \\
\text { deployed and retracted }\end{array}$ & 32.2 \\
\hline
\end{tabular}




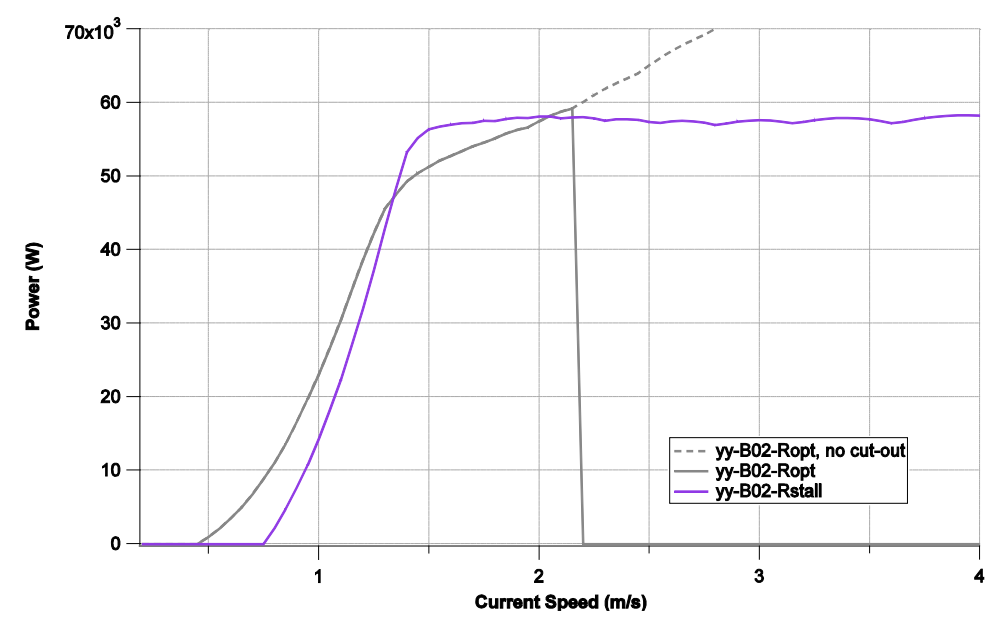

Figure III.4. Power curves of yy-B02-Rcs,opt and yy-B02-Rstall, stall-regulated rotors with a rated power of $60 \mathrm{~kW}$. The dashed line for the yy-B02-Rcs,opt represents power production without a prescribed cut-out.

Figure III.4 shows the power curves of rotors yy-B02-Rcs,opt and yy-B02-Rcs,stall, which both use the yyb07cn family of hydrofoils. The yy-B02-R $c s, o p t$ is optimized for energy capture with cut-out upon reaching rated power. The $y \mathrm{y}-\mathrm{B} 02-\mathrm{R}$ cs,stall has a negative blade incidence angle that pushes the rotor into stall as it nears rated power. Unlike the power curve of the e-B02-Rcs,opt, Region III is almost flat, exhibiting almost ideal behavior. This is a result of the stall characteristics of the yyb07cn-180 hydrofoil, which retains more lift in stall.

As presented in Table III-2, with a Rayleigh distribution with a $0.87 \mathrm{~m} / \mathrm{s}$ mean speed, the capacity factors of the yy-B02-Rcs,opt and yy-B02-Rcs,stall are $31.3 \%$ and $25.9 \%$, respectively. The yy-B02$\mathrm{R} c s, o p t$ has a $0.44 \%$ higher capacity factor (0.1 percentage points) than the e-B02-R $c s, o p t$. The yyB02-Rcs,opt must cut-out at approximately $2.2 \mathrm{~m} / \mathrm{s}$, but this has little impact on its overall energy capture because such high current speeds are infrequent. In comparison, the energy capture of the yy-B02-Rcs,stall is $17.3 \%$ lower. This is reflected in the power curves in Figure III.4. The yy-B02$\mathrm{R} c s, o p t$ produces more power between approximately $0.5 \mathrm{~m} / \mathrm{s}$ and $1.3 \mathrm{~m} / \mathrm{s}$. As evident in the current speed distributions in Figure III.1, the rotor operates frequently within this speed range. The reduced performance is due to the blade incidence angle of the yy-B02-Rcs,stall. At $8^{\circ}$, it produces favorable power regulation characteristics, but is very far from optimal power production (which is nominally at $0^{\circ}$ ).

It is important to recall that the performance calculations presented here are based on computational modeling of hydrodynamic performance. Both XFOIL (couple viscous-inviscid method) and RaNS are generally very accurate in the linear portions of the life curve, but less so at and near stall. It is prudent to verify performance near stall via experiment (i.e., wind tunnel or water tunnel).

Figure III. 5 shows the power curves of rotors with the yyb07cn hydrofoils with microtabs incorporated into the design. yy $\mu \mathrm{t}-\mathrm{B} 02-\mathrm{R} c s, o p t-d e p$ (the red line) represents a rotor with energy capture optimized with microtabs deployed. Recall that this would be normal operation for the BRT'T, with microtabs deploying on either side of the blade as current direction switches. The capacity factor in this case is 31.5\%, 0.64\% higher than without microtabs and $0.96 \%$ higher than with simple elliptical hydrofoils. yy $\mu \mathrm{t}-\mathrm{B} 02-\mathrm{R} c s$, fail (the green line) represents a failure mode in which the microtabs are stuck in a retracted position. Because the turbine was optimized to run with the 
microtabs deployed, performance drops with a capacity factor of $29.9 \%, 5.1 \%$ less than the case with the microtabs correctly deployed.

In the yy $\mu \mathrm{t}-\mathrm{B} 02-\mathrm{R} c s$, opt case (the grey line), the microtabs are employed for load control, delaying the need to cut-out. The microtabs are deployed up to $1.65 \mathrm{~m} / \mathrm{s}$. Note that this portion of the curve does not follow that of yy $\mu \mathrm{t}-\mathrm{B} 02-\mathrm{Rcs}, \rho_{\mathrm{t}}$-dep (red) since they are optimized for different operating conditions; although the blades are the same, the blade incidence angles and rotor speeds are slightly different. Above $1.65 \mathrm{~m} / \mathrm{s}$, the microtabs are retracted, keeping power output below rated. Operation then continues to $2.35 \mathrm{~m} / \mathrm{s}$ before cutting-out. This strategy of microtab usage yields a capacity factor of $32.2 \%, 2.2 \%$ higher than using microtabs only for current direction switching (yy $\mu \mathrm{t}-\mathrm{B} 02-\mathrm{R} c s, o p t-d e p$ ), 2.9\% higher than not using microtabs at all (yy-B02-Rcs,opt), and 3.1\% higher than with simple elliptical hydrofoils (e-B02-Rcs,opt).

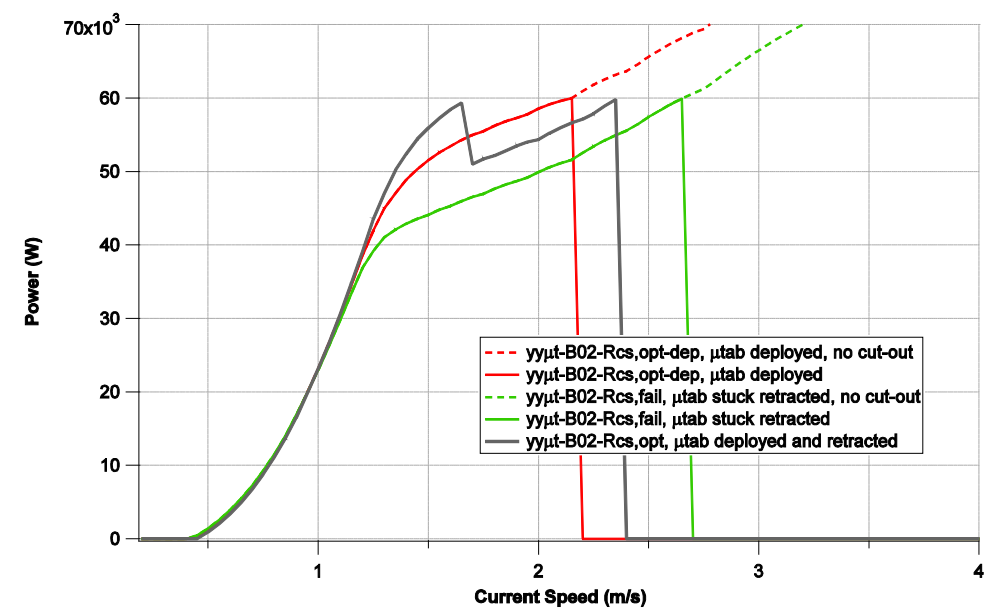

Figure III.5. Power curves of yy $\quad$ Pt-B02-Rcs,opt-dep (optimized for energy capture with microtabs deployed), yy $\mu \mathrm{t}-$ B02-Rcs,fail (optimized for energy capture with microtabs deployed, but with microtabs stuck in the retracted position), and yy $\mu \mathrm{t}-\mathrm{B} 02-\mathrm{R} c s, o p t$ (optimized for energy capture with microtabs optimally deployed and retracted). These are stallregulated rotors with a rated power of $60 \mathrm{~kW}$. The dashed lines represent performance without prescribed cut-out. 
Table III-3 lists the differences in energy capture between various constant speed rotor configurations, along with associated changes in annual energy revenue for a range of energy prices. To maintain the same cost of energy, increases in capital costs and, more importantly in MHK applications, O\&M costs, must be offset by increased energy revenue. The yy hydrofoils without microtabs are an obvious improvement over elliptical hydrofoils, as they provide an energy capture benefit at essentially no additional cost. The addition of optimally used microtabs (yy $\mu \mathrm{t}-\mathrm{B} 02-\mathrm{R} c s$ ) provides a 3.67\% increase in energy and revenue capture, but introduces significant system complexity which increases both capital and O\&M costs. In terms of absolute values, the revenue increase is relatively small, $\$ 1,543 /$ year at $8 \phi / \mathrm{kWh}$; however, this is for a small $60 \mathrm{~kW}$ turbine. Microtabs may be better suited for larger turbines where they would yield larger revenue increases.

Table III-3.Comparison of energy capture/revenue between various constant speed rotor configurations. Changes in annual revenue are presented for a range of energy prices.

\begin{tabular}{|c|c|c|c|c|c|c|}
\hline & & & & & & \\
\hline & & & Chat & e in Annu & Energy Re & nue (\$) \\
\hline & & & & Ene & Price & \\
\hline Configuration & Compared to... & $\begin{array}{l}\text { Change in } \\
\text { Energy } \\
\text { Capture or } \\
\text { Revenue } \\
\text { (\%) }\end{array}$ & $8 \$ / k W h$ & $12 థ / \mathrm{kWh}$ & $15 \$ / k W h$ & $20 \$ / k W h$ \\
\hline yy-B02-Rcs, opt & e-B02-Rcs,opt & $0.45 \%$ & 188 & 282 & 352 & 470 \\
\hline yy-B02-Rcs, stall & yy-B02-Rcs,opt & $-17.23 \%$ & $-7,248$ & $-10,873$ & $-13,591$ & $-18,121$ \\
\hline yу $\mu t-B 02-R c s, o p t-d e p$ & e-B02-Rcs,opt & $1.05 \%$ & 442 & 662 & 828 & 1,104 \\
\hline yy $\mu \mathrm{t}-\mathrm{B} 02-\mathrm{Rcs}$, opt-dep & yy-B02-Rcs,opt & $0.60 \%$ & 252 & 379 & 473 & 631 \\
\hline yy & уy $\mu t-B 02-R c s, o p t-d e p$ & $-5.21 \%$ & $-2,193$ & $-3,289$ & $-4,111$ & $-5,481$ \\
\hline yy & yy-B02-Rcs,opt & $-4.64 \%$ & $-1,953$ & $-2,930$ & $-3,662$ & $-4,883$ \\
\hline yy $\mu$ t-B02-Rcs, opt & уy $\mu t-B 02-R c s, o p t-d e p$ & $3.05 \%$ & 1,283 & 1,924 & 2,405 & 3,207 \\
\hline yy $\mu \mathrm{t}-\mathrm{B} 02-\mathrm{Rcs}, \mathrm{opt}$ & yy-B02-Rcs,opt & $3.67 \%$ & 1,543 & 2,314 & 2,893 & 3,857 \\
\hline yy $\mu \mathrm{t}-\mathrm{B} 02-\mathrm{Rcs}, \mathrm{opt}$ & e-B02-Rcs,opt & $4.13 \%$ & 1,738 & 2,607 & 3,258 & 4,344 \\
\hline
\end{tabular}




\section{COMPARISON OF ACCOMPLISHMENTS WITH INITIAL GOALS AND OBJECTIVES}

The primary goal of this project was to bring application of microtabs on BRT'T rotors to TRL 3. This was largely successful, although the path taken was different than initially conceived.

The most significant deviation was the design and development of the yy foils. The yy foils offer improved performance over existing bidirectional foils without any of the complexities of an active system. However, their bluff trailing edges complicated their design. The separated flow at the trailing edge precluded the use of inverse foil design tools such as XFOIL. For the same reason, the viscous-inviscid interaction solver of XFOIL could not be used for analysis. The foil design was therefore primarily conducted through direct design (i.e., geometry manipulation) and RaNS CFD, a process intensive in both time and computational resources.

The yy foils were essential to the application of microtabs on BRTT rotors. As initially conceived, the primary hydrofoil for the study was to be an ellipse or something very similar. Microtabs on the $18 \%$ ellipse were found to be ineffective; performance actually decreased. On the yy foils, microtabs improved performance as expected, increasing $1 / \mathrm{d}$. They also had the unexpected benefit of improving the linearity of the lift curve slope and attenuating the lift drop-off beyond stall.

The yy foil development came at the expense of some of the project's secondary goals, which focused on the study of microtabs for active load alleviation on BRTT rotors. However, before engaging in such study, it would be valuable to first conduct experimental (i.e., wind or water tunnel) testing of the yy hydrofoils to verify stall and near-stall behavior.

Summaries of accomplishments and items for further study (both newly and previously identified) are provided below.

\section{IV.1. SUMMARY OF ACCOMPLISHMENTS AND KEY FINDINGS}

- The use of microtabs on a BRT'T rotor to improve performance of bidirectional hydrofoils was demonstrated via computational modeling.

- A series of bidirectional hydrofoils, dubbed the yy foils, were designed. These foils exhibited better performance than the simple ellipse. For example, the yyb07cn-180 had 14.7\% higher $(\mathrm{l} / \mathrm{d})_{\max }$ than an ellipse of equal thickness. The yyb07cn family also had higher $\mathrm{c}_{\mathrm{p} \text {, min }}$ than equivalently thick ellipses, indicating less susceptibility to cavitation.

- Microtabs were not always effective on bluff trailing edge foils. In particular, hydrodynamic performance of an 18\% thick ellipse decreased with the addition of a microtab. For bluff trailing edge foils, the use of microtabs must be considered during the foil design/selection process.

- A series of variable speed and constant speed rotors were developed with the yyb07cn family of hydrofoils. Comparable rotors were also developed with elliptical foils. The constant speed yyb07cn rotor (yy-B02-Rcs,opt) captured $0.45 \%$ more energy than the equivalent rotor with ellipses (e-B02-R $c s, o p t)$. With microtabs deployed (yy $\mu \mathrm{t}-\mathrm{B} 02-\mathrm{R} c s, o p t)$, the energy capture increase over the rotor with ellipses was $1.05 \%$. 


\section{IV.2. ONGOING AND FUTURE WORK}

- The yy hydrofoils, with and without microtabs, should be tested experimentally in a wind or water tunnel. Verification of hydrodynamic behavior at and near stall would be particularly valuable.

- The presence of the retracted, forward microtab on the suction surface may detrimentally affect performance, causing premature boundary layer transition or separation, or cavitation inception. The impact on the boundary layer can be studied computationally or experimentally. Cavitation study might be best conducted via experiment.

- The benefits of microtabs need to be further quantified to establish upper bounds for the microtab system cost. The benefits should include:

- The use of microtabs for active load control to mitigate load excursions from shear, tower shadowing, yaw error, and surging current. The resulting reduction in fatigue damage should reduce O\&M costs and extend turbine life, improving levelized cost of energy.

- Microtabs simultaneously deployed at multiple positions on a foil may provide a braking capability. 


\section{DEVELOPED PRODUCTS}

The yy foils were the primary new product developed in this study. We have withheld from beginning a patent application for the yy foils until a licensee has been identified. This is necessitated by our patent filing process, which requires that a declaration of invention first be made and then a licensee found within one year. If no licensing occurs within the year, the invention/patent is abandoned.

We have discussed this study, including the yy foils, with Sandia National Laboratories. The yy foil geometries have not yet been released. 


\section{REFERENCES}

[1] “Economic Viability of a Simple Tidal Stream Energy Capture Device," UK DTI, TP/3/ERG/6/1/15527/REP URN 07/575, 2007.

[2] "Voith Hydro Ocean Current Technology marketing material." [Online]. Available: http://www.voithhydro.com/media/t331_Ocean_Current_Technologies_72dpi.pdf. [Accessed: 01-Jun-2010].

[3] M. Drela, "XFOIL: An Analysis and Design System for Low Reynolds Number Airfoils," presented at the Conference on Low Reynolds Number Airfoil Aerodynamics, University of Notre Dame, 1989.

[4] P. G. Buning, D. C. Jesperson, T. H. Pulliam, and et al, "OVERFLOW User's Manual, Version 1.8aa." NASA Langley Research Center, Apr-2003.

[5] "NWTC Design Codes (WT_Perf by Andrew Platt)." [Online]. Available: http://wind.nrel.gov/designcodes/simulators/wtperf/. [Accessed: 20-May-2013].

[6] "NWTC Computer-Aided Engineering Tools (AirfoilPrep by Dr. Craig Hansen)." [Online]. Available: http://wind.nrel.gov/designcodes/preprocessors/airfoilprep/. [Accessed: 20-May2013].

[7] W. Chan, S. Rogers, S. Pandya, D. Kao, P. Buning, R. Meakin, D. Boger, and S. Nash, "User's Manual for Chimera Grid Tools, Version 2.1," NASA Ames Research Center, Mar. 2010.

[8] S. F. Hoerner, Fluid-dynamic lift: practical information on aerodynamic and bydrodynamic lift. Brick Town, N.J.: L.A. Hoerner, 1975.

[9] D. S. Thompson and GB, "United States Patent: 8115329 - Water turbine with bi-symmetric airfoil," 811532914-Feb-2012.

[10] S. Gooch, J. Thomson, B. Polagye, and D. Meggitt, "Site characterization for tidal power," in OCEANS 2009, MTS/IEEE Biloxi - Marine Technology for Our Future: Global and Local Challenges, 2009, pp. 1-10. 